ARTICLE

\title{
GABA signalling modulates stomatal opening to enhance plant water use efficiency and drought resilience
}

Bo Xu (1) 1,2, Yu Long1,2, Xueying Feng (10) 1,2, Xujun Zhu (10) 1,3, Na Sai (1) 1,2, Larissa Chirkova ${ }^{2,4}$, Annette Betts ${ }^{5}$, Johannes Herrmann ${ }^{6}$, Everard J. Edwards (10) ${ }^{5}$, Mamoru Okamoto (1) ${ }^{2,4}$, Rainer Hedrich (1) ${ }^{6}$ \& Matthew Gilliham (1) 1,2凶

The non-protein amino acid $\gamma$-aminobutyric acid (GABA) has been proposed to be an ancient messenger for cellular communication conserved across biological kingdoms. GABA has welldefined signalling roles in animals; however, whilst GABA accumulates in plants under stress it has not been determined if, how, where and when GABA acts as an endogenous plant signalling molecule. Here, we establish endogenous GABA as a bona fide plant signal, acting via a mechanism not found in animals. Using Arabidopsis thaliana, we show guard cell GABA production is necessary and sufficient to reduce stomatal opening and transpirational water loss, which improves water use efficiency and drought tolerance, via negative regulation of a stomatal guard cell tonoplast-localised anion transporter. We find GABA modulation of stomata occurs in multiple plants, including dicot and monocot crops. This study highlights a role for GABA metabolism in fine tuning physiology and opens alternative avenues for improving plant stress resilience.

\footnotetext{
${ }^{1}$ Plant Transport and Signalling Lab, ARC Centre of Excellence in Plant Energy Biology, Waite Research Institute, Glen Osmond, SA, Australia. ${ }^{2}$ School of Agriculture, Food and Wine, Waite Research Precinct, University of Adelaide, Glen Osmond, SA, Australia. ${ }^{3}$ College of Horticulture, Nanjing Agricultural University, Nanjing, China. ${ }^{4}$ ARC Industrial Transformation Research Hub for Wheat in a Hot and Dry Climate, Waite Research Institute, University of Adelaide, Glen Osmond, SA, Australia. ${ }^{5}$ CSIRO Agriculture \& Food, Glen Osmond, SA, Australia. ${ }^{6}$ Institute for Molecular Plant Physiology and Biophysics, University of Würzburg, Würzburg, Germany. ${ }^{凶}$ email: matthew.gilliham@adelaide.edu.au
} 
T he regulation of stomatal pore aperture is a key determinant of plant productivity and drought resilience, and profoundly impacts climate due to its influence on global carbon and water cycling ${ }^{1-3}$. The stomatal pore is delineated by a guard cell pair. Fine control of ion and water movement across guard cell membranes, via transport proteins, determines cell volume and pore aperture following opening and closing signals such as light and dark $2,4,5$ (Fig. 1). Due to their critical roles and their ability to respond to and integrate multiple stimuli, stomatal guard cells have become a preeminent model system for investigating plant cell signalling ${ }^{6}$ resulting in the elucidation of many critical pathways involved in plant biotic and abiotic stress tolerance $^{7-9}$.

GABA signalling in mammals relies upon receptor-mediated polarization of neuronal cell membranes ${ }^{10,11}$. Speculation that GABA could be a signal in plants is decades old ${ }^{12}$, but a definitive demonstration of its mode of action remains elusive. GABA production in plants is upregulated by stress ${ }^{13,14}$. It is synthesised in the cytosol via the GABA shunt pathway, bypassing two stressinhibited reactions of the mitochondrial-based tricarboxylic acid (TCA) cycle ${ }^{15,16}$. GABA is therefore well known as a stressinduced plant metabolite that is fed back into the mitochondrial TCA cycle to sustain cellular energy production ${ }^{12,17}$. The discovery that the activity of aluminium-activated malate transporters (ALMTs) can be regulated by $\mathrm{GABA}^{18}$ represents a plausible mechanism by which GABA signals could be transduced in plants, providing a putative-but unproven - novel signalling link between primary metabolism and physiology ${ }^{19}$. Stomatal guard cells contain a number of ALMTs that impact stomatal movement and transpirational water $\operatorname{loss}^{20-22}$. Therefore, stomatal guard cells represent an ideal system to test whether GABA signalling occurs in plants.

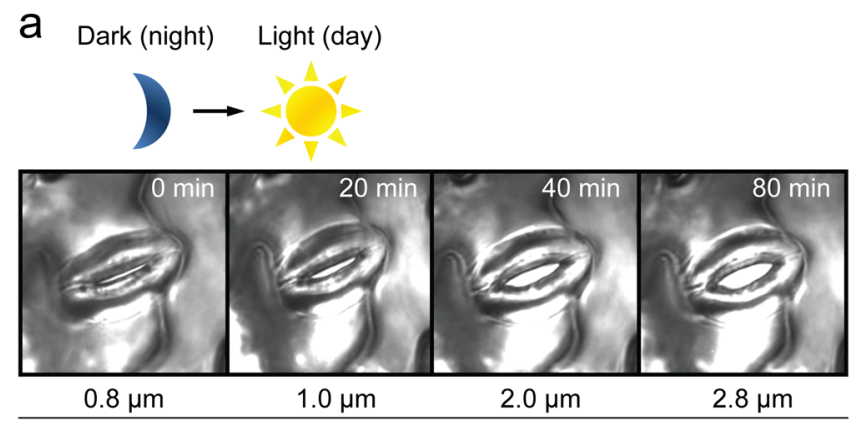

Stomatal aperture width

b
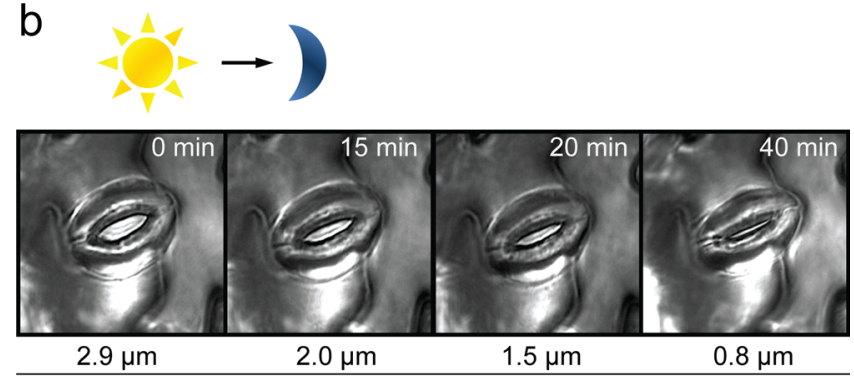

Stomatal aperture width

Fig. 1 Guard cells respond to light signals. a, b Time course of lightinduced stomatal opening (a) and dark-induced stomatal closure (b) with actual stomatal aperture width indicated below; dark-to-light transition mimics night-to-day transition which opens stomatal pores (a) and light-todark transition mimics day-to-night transition which closes stomatal pores (b), light intensity $150 \mu \mathrm{mol} \mathrm{m}-2 \mathrm{~s}^{-1}$.
Significantly, here, we show that GABA does not initiate changes in stomatal pore aperture, rather it antagonises changes in pore size, which differentiates it from many of the signals known to regulate stomatal aperture ${ }^{3-8}$. Specifically, we find that GABA concentration increases under a water deficit and this reduces stomatal opening in an ALMT9-dependant manner. The anion channel ALMT9 is a major pathway for mediating anion uptake into the vacuole during stomatal opening ${ }^{21}$; GABA signal transduction via ALMT9 leads to reduced transpirational water loss, increased water use efficiency (WUE) and improved drought resilience. As such, even though guard cell signalling is relatively well defined ${ }^{6,23}$, this study has been able to uncover another pathway regulating plant water loss. Furthermore, by revealing a mechanism by which GABA acts in stomatal guard cells, we demonstrate that GABA is a legitimate plant signalling molecule ${ }^{16}$.

\section{Results}

GABA antagonises both stomatal pore opening and closure in epidermal peels, but only opening in leaf feeding experiments. To validate whether GABA is a physiological signal that modulates stomatal pore aperture, our initial experiments used excised Arabidopsis thaliana epidermal peels where stomatal guard cells are directly accessible to a chemical stimuli ${ }^{8,24-26}$. When exogenous GABA or its analogue muscimol ${ }^{14}$ were applied under constant light or dark conditions, neither elicited a change in stomatal aperture (Fig. 2a, b; Supplementary Fig. 1a, b). Interestingly though, we found that both compounds suppressed lightinduced stomatal opening and dark-induced stomatal closure (Fig. 2a, b; Supplementary Fig. 1a, b). We then fed intact leaves with an artificial sap solution through the detached petiole with or without the addition of GABA or muscimol and examined whether this affected gas exchange rates. We found, in the GABA and muscimol fed leaves, that the increase in water loss (transpiration) stimulated by a dark-to-light transition was dampened compared to leaves fed just the artificial sap solution due to reduced stomatal conductance (Fig. 2c; Supplementary Figs. 1c, d and 2a). This is consistent with the reduced extent of stomatal opening that we observed in epidermal peels in the presence of GABA or muscimol upon a dark-to-light transition (Fig. 2b; Supplementary Fig. 1a). The gas exchange values of fed leaves were used to calculate instantaneous intrinsic WUE (iWUE) and WUE (ratios of carbon gained through photosynthesis per unit of water lost), which are key traits underpinning drought tolerance in plants ${ }^{27}$, and both values were greater (i.e. improved) in GABA fed leaves (Fig. 2d; Supplementary Fig. 2a-c).

GABA is a universal stomatal behaviour modifier. To examine whether GABA or muscimol can modulate stomatal aperture beyond the response to light and dark, we examined their impact on a range of opening and closing signals using epidermal peels of Arabidopsis. We found both GABA and muscimol inhibited abscisic acid- (ABA, $2.5 \mu \mathrm{M}$ ) or $\mathrm{H}_{2} \mathrm{O}_{2}$-stimulated stomatal closure and coronatine-induced opening (Supplementary Fig. 3a, c, e, f $)^{8,28}$. However, stomatal pores were fully closed in response to high concentrations of ABA $(25 \mu \mathrm{M})$ (Supplementary Fig. $3 \mathrm{~b}, \mathrm{~d}$ ) or exogenous calcium in the presence of GABA or muscimol (Supplementary Fig. 3g), which indicated stomatal closure could occur in epidermal peels in the presence of GABA when the closing signal was of sufficient magnitude.

We tested whether our results could be explained by GABA or muscimol treatment permanently locking guard cells in a closed (or open) state and preventing further change in stomatal pore aperture, which would argue against GABA being a physiological signal. We did this by incubating epidermal peels in GABA or 
a

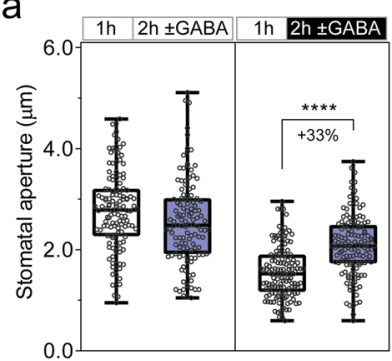

b
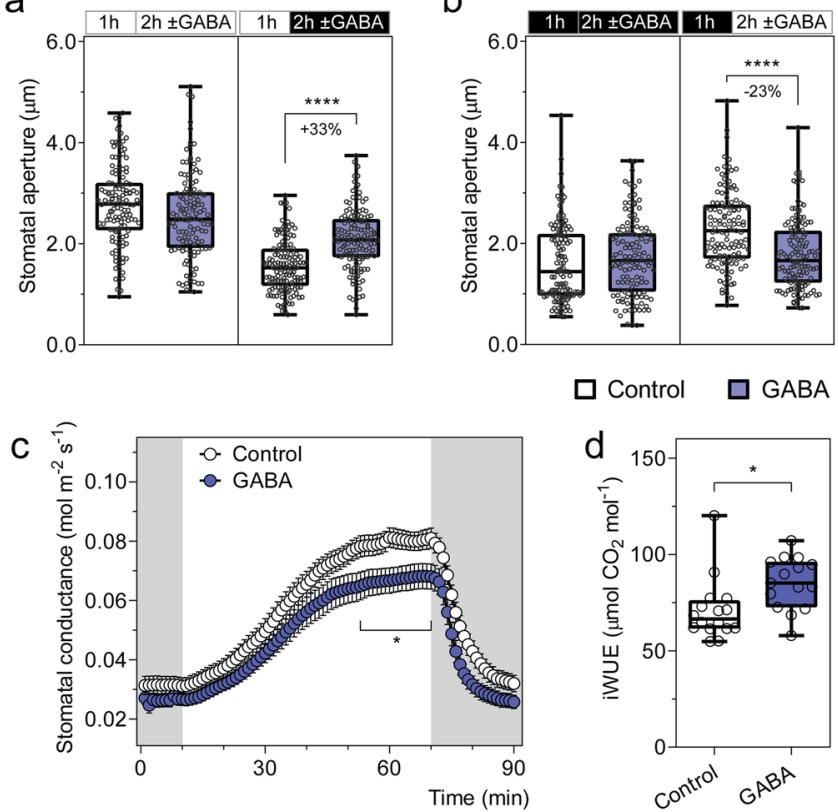
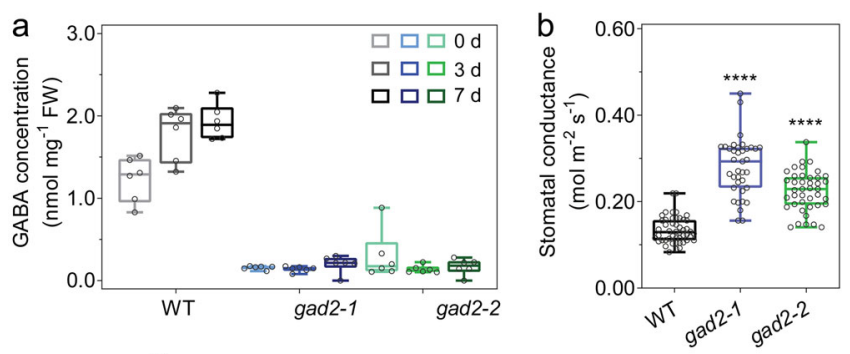

C

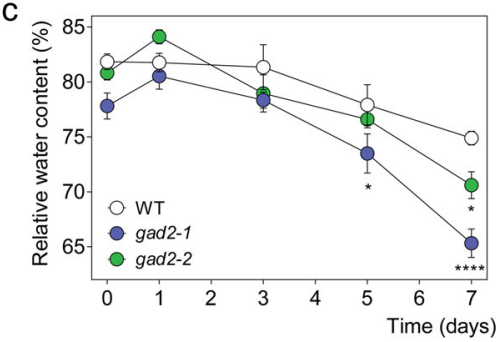

Fig. 3 Leaf GABA concentration regulates transpiration. a Leaf GABA concentration of 5-6-week-old A. thaliana wild-type (WT), gad2-1 and gad22 plants following drought treatment for 0,3 and 7 days, $n=6$. b Stomatal conductance of Arabidopsis WT, gad2-1 and gad2-2 plants determined using an AP4 porometer; $n=48$ for WT, $n=37$ for gad2-1 and $n=41$ for gad2-2, data collected from three independent batches of plants. c Relative leaf water content of WT, gad2-1 and gad2-2 plants following drought treatment for $0,1,3,5$ and 7 days, $n=6$. All data are plotted with box and whiskers plots: whiskers plot represents minimum and maximum values, and box plot represents second quartile, median and third quartile $(\mathbf{a}, \mathbf{b})$, or data are represented as mean \pm s.e.m (c); statistical difference was determined using two-way ANOVA $(\mathbf{a}, \mathbf{c})$ or one-way ANOVA (b): ${ }^{\star} P<0.05$ and ${ }^{\star \star \star \star} P<00.0001$.

epidermal strips attenuated stomatal responses of other plant species to light or dark transitions, including the dicot crops Vicia faba (broad bean), Glycine max (soybean) and Nicotiana benthamiana (tobacco-relative) and the monocot Hordeum vulgare (barley) (Supplementary Fig. 5). The widespread inhibition of stomatal pore aperture changes suggests that GABA has the potential to be a universal 'brake' on stomatal movement in plants, including valuable crops.

detached leaves from 5- to 6-week-old $A$ thaliana wild-type plants was recorded using a LI-COR LI-6400XT in response to dark (shaded region) and $200 \mu \mathrm{mol} \mathrm{m} \mathrm{m}^{-2} \mathrm{~s}^{-1}$ light (white region), fed with artificial xylem sap solutions $\pm 4 \mathrm{mM}$ GABA. d iWUE efficiency of detached leaves was calculated as the ratio of photosynthetic rate (Supplementary Fig. $2 \mathrm{~b}$ ) versus stomatal conductance (c); $n=16$ independent leaves for control and $n=15$ independent leaves for GABA, data collected from three different batches of plants (c, d). All data are plotted with box and whiskers plots: whiskers plot represents minimum and maximum values, and box plot represents second quartile, median and third quartile $(\mathbf{a}, \mathbf{b}, \mathbf{d})$, or data are represented as mean \pm s.e.m (c); statistical difference was determined by two-way ANOVA (a, b), or two-sided Student's $t$ test $(\mathbf{c}, \mathbf{d}),{ }^{\star} P<0.05$ and ${ }^{\star \star \star \star} P<$ 0.0001

muscimol, then removing this treatment and performing a light or dark transition. As would be expected from viable cells, after removal of the GABA or muscimol treatment, we found that stomatal guard cells responded to a light treatment by opening the pores (Supplementary Fig. 4a, b) or to a dark treatment by closing pores (Supplementary Fig. 4c, d). Collectively, these data again indicate that GABA signals would likely act to modulate stomatal aperture in the face of a stimulus rather than stimulating a transition itself.

To test whether GABA is a universal modulator of stomatal control, we explored whether GABA or muscimol treatment of
GABA accumulation in guard cells contributes to the regulation of transpiration and drought performance. Stomatal control is explicitly linked with the regulation of plant water loss, the stomatal aperture, the greater the water loss of plants, the poorer the survival of plants under a limited water supply, as excessive water use by the plant diminishes the availability of stored soil water. The observation that the stress-induced metabolite $\mathrm{GABA}^{13}$ reduces plant water loss and improves WUE (Fig. 2d; Supplementary Fig. 2c)-key factors underpinning drought tolerance ${ }^{27}$-implicates GABA as novel signal regulating plant drought resilience. Therefore, to examine the hypothesis that endogenous GABA concentration increases under a water deficit and acts as a signal, we first determined whether we could replicate the previously reported increases in GABA accumulation under drought ${ }^{13,14,29}$ (Fig. 3; Supplementary Fig. 6). In wild-type plants, a drought treatment was applied by withholding watering, which resulted in the gradual depletion of soil gravimetric water and a reduction in leaf relative water content (RWC) (Supplementary Fig. 6a, b). We found that GABA accumulation in drought stressed leaves increased by $35 \%$ compared to that of well-watered leaves (water versus drought at which impacts the survival of plants under drought ${ }^{7}$; the wider 
7 days: $1.07 \pm 0.08$ versus $1.44 \pm 0.11 \mathrm{nmol} \mathrm{mg}^{-1} \mathrm{FW}$ ) (Supplementary Fig. 6c).

To investigate whether GABA has a role during drought, we obtained Arabidopsis T-DNA insertional mutants for the major leaf GABA synthesis gene, Glutamate Decarboxylase 2 (GAD2) ${ }^{29}$. Both gad2-1 and gad2-2 had $>75 \%$ less GABA accumulation in leaves than in wild-type plants, whilst GABA concentrations in roots were unchanged (Fig. 3a; Supplementary Fig. 6d-f). Furthermore, leaves of gad2 plants did not accumulate additional GABA under drought conditions unlike wild-type controls where GABA increased by $45 \%$ after 3 days, and was maintained at this elevated level after 7 days of drought (Fig. 3a). Under standard conditions, both gad2 mutant lines exhibited greater stomatal conductance and wider stomatal pores than wild-type plants (Fig. 3b; Supplementary Fig. 6g), whereas stomatal density was identical to wild type (Supplementary Fig. 6h). The application of exogenous GABA to gad2 leaves inhibited stomatal pore aperture changes in response to light treatments (Supplementary Fig. 6i, j), indicating that gad2 stomata would be competent in a GABA response if sufficient GABA was present. Furthermore, the aperture of GABA pre-treated $g a d 2$ stomata after a dark-to-light transition were statistically insignificant from non-GABA treated wild-type stomata (Supplementary Fig. 6j), which is consistent with GABA playing a role in modulating opening of wild-type stomata under non-stressed conditions. It has been shown previously that both GAD2 transcription and GABA accumulation exhibit diurnal regulation; GABA usually peaks at the end of the dark cycle prior to stomatal opening and reaches a minimum when stomatal conductance is at its maximum near subjective mid-day ${ }^{30}$. However, during stress, both GAD2 transcript abundance and GABA accumulation remain high ${ }^{30}$. This suggests GABA may further minimise stomatal opening under stress and contribute to drought tolerance.

Under drought, the leaf RWC of gad2 plants lowered more quickly than in wild type (Fig. 3c). Transcriptional profiles of key ABA-marker gene (RD22) and GABA-related genes (other than $G A D 2$ ) were similar in wild type and gad2 lines, although $R D 29 A$ was significantly higher in gad2-1 than wild type and gad2-2 on day 0 and day 7 of the drought treatment (Supplementary Fig. 7), which is consistent with the lower RWC of gad2-1 after 7 days (Fig. 3c). These results confirm that GAD2 is critical for leaf GABA production under stress, and suggests that GABA itself may regulate plant water loss and drought tolerance ${ }^{29}$.

Histochemical staining corroborated that GAD2 is highly expressed in leaves, particularly in guard cells ${ }^{29}$ (Supplementary Fig. $8 \mathrm{a}, \mathrm{b}$ ). GAD2 is a cytosolic enzyme ${ }^{31}$; to examine if cytosolic GABA biosynthesis within the guard cell was sufficient to modulate transpiration we expressed-specifically in the guard cell $^{32}$ - a constitutively active form of GAD2 (GAD2 $\triangle$ ) that has a C-terminal autoinhibitory domain removed ${ }^{31,33}$ (Fig. 4a). This led to a large increase in leaf GABA accumulation (Fig. 4b) and to complementation of the steady-state stomatal conductance and aperture phenotypes of gad2 plants to wild-type levels (Fig. 4c; Supplementary Fig. 8c, d). At the same time, no change in stomatal density or leaf ABA accumulation was detected under standard conditions (Supplementary Fig. 8e, f), suggesting the complementation of the gad2 phenotype was due to the restoration of GABA synthesis in the guard cell. Other phenotypes restored to wild-type levels by guard cell-specific expression of GAD2 $\triangle$ included the exaggerated stomatal opening and closure kinetics and decreased instantaneous iWUE/WUE of gad2-1 (Fig. 4d-f; Supplementary Fig. 8g-i). The drought sensitivity of gad2, compared to wild type, was also abolished by guard cellspecific expression of GAD2A (Fig. 4g, h). This demonstrates GABA synthesis in guard cells was sufficient to modulate stomatal movement, regulate water loss and improve drought resilience.
To examine whether GABA metabolism can be modulated to improve drought resilience beyond wild-type levels, GAD2 $\triangle$ was expressed specifically in the guard cells of wild-type Arabidopsis plants (Fig. 5a), this resulted in leaf GABA concentrations being increased to beyond wild-type levels (Fig. 5b). The steady-state stomatal conductance of the GABA overproducing transgenic plants in standard and drought conditions was lowered compared to wild-type plants (Fig. $5 \mathrm{c}$ ). Consistent with this, the plants overexpressing GAD2 $\triangle$ in the wild-type background maintained higher leaf RWC than wild-type plants after 10 days of drought treatment (Fig. 5d, e). Furthermore, a greater percentage of plants overexpressing GAD2 $\Delta$ in the wild-type background survived following re-watering after a 12-day drought treatment (Supplementary Fig. 9). As such, we show here that GABA overproduction can reduce water loss and improve drought resilience.

Guard cell cytosolic GABA modulates stomatal movement and drought resilience. Our data show that although guard cell synthesised GABA can rescue the gad2 phenotype, it is clear that exogenously applied GABA can also modulate stomatal movement (e.g. Fig. 2 for wild type or Supplementary Fig. 6i, j for $\operatorname{gad} 2$ ). It is known that GABA can pass the membrane through a variety of transporters ${ }^{34-36}$, so it is unclear whether the site of guard cell GABA action is from the apoplast or cytoplasm. We expressed GAD2 2 specifically in the spongy mesophyll ${ }^{37}$, adjacent to the abaxial stomatal layer, to test whether it could complement gad2 (Supplementary Fig. 10a, b). This resulted in a significant increase in leaf GABA, but no change in stomatal conductance (Supplementary Fig. 10c, d). As such, unlike guard cell-specific expression, GAD2 $\triangle$ in the spongy mesophyll was insufficient to complement the gad2-1 phenotype.

To further probe the role of guard cell synthesised GABA, we expressed full-length GAD2 under the guard cell-specific promoter (gad2-1/GC1::GAD2) (Supplementary Fig. 11a). This form of GAD2 requires activation by $\mathrm{Ca}^{2+} /$ calmodulin or low $\mathrm{pH}$ to synthesise $\mathrm{GABA}^{14}$. Interestingly, guard cell-specific expression of full-length GAD2 failed to complement the high stomatal conductance of the gad2-1 line to wild-type levels under standard conditions, whereas its constitutive expression (driven by pro35S-CAMV) did (Supplementary Fig. 11b, f, g). Under drought, the gad2-1/GC1::GAD2 lines increased GABA production, reduced their stomatal conductance significantly more than that of gad2-1 plants and had a comparable leaf RWC to wild-type plants following 5 days of drought (Supplementary Fig. $11 \mathrm{c}-\mathrm{e}$ ). This suggests that activation of fulllength GAD2 via its regulatory domain ${ }^{31}$ is important in stimulating GABA production under drought in guard cells.

We extended our investigation of GABA's site of action through an epidermal peel experiment. We compared the effects of exogenously applied muscimol or muscimol-BODIPY, a muscimol molecule conjugated with a BODIPY fluorophore, which is active against GABA targets in plants and animals, but lacks cell-membrane permeability ${ }^{38,39}$. We found that unlike muscimol, membrane impermeable muscimol-BODIPY was unable to inhibit stomatal opening or closure (Supplementary Fig. 12). This result-alongside the differential effects of gad2 complementation by full-length GAD2 when expressed constitutively or solely in the guard cell (Supplementary Fig. 11a-e) - provides further evidence that GABA is likely to pass the plasma membrane and that it acts from the cytosol, consistent with our feeding assays (e.g. Fig. 2c). Collectively, the data in this section demonstrate that guard cell-specific cytosolic GABA accumulation is sufficient and necessary for controlling stomatal aperture and transpiration under drought, but suggests a role for other cell types in fine-tuning GABA signals under standard conditions. 
a
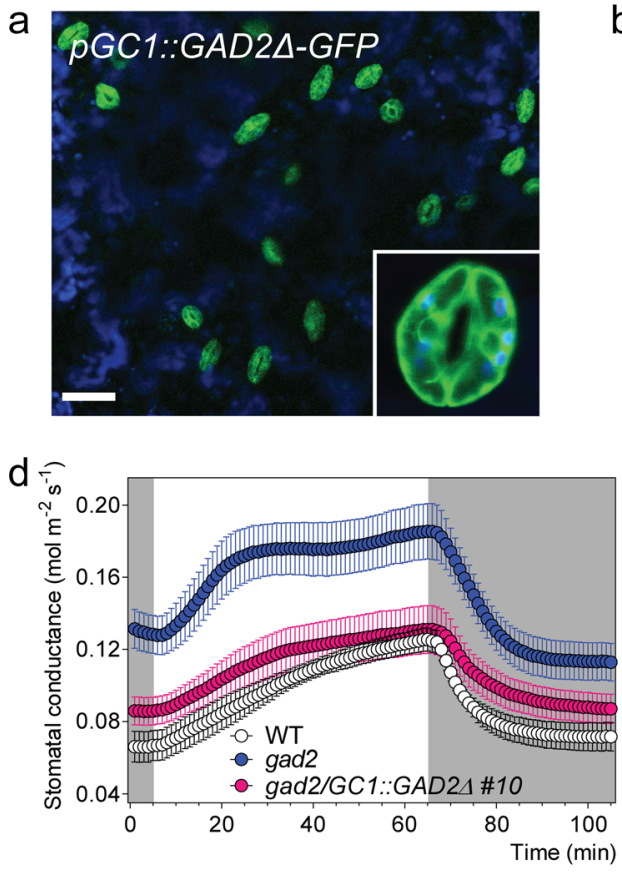

g

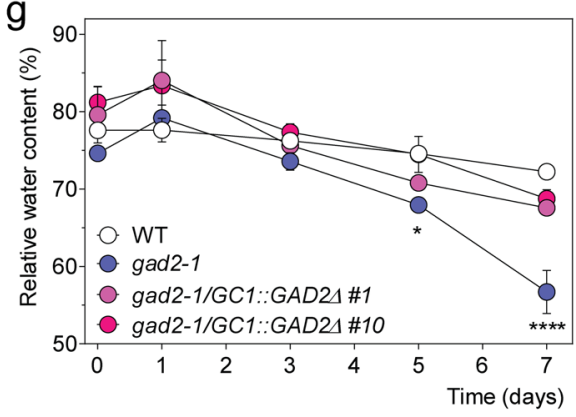

b

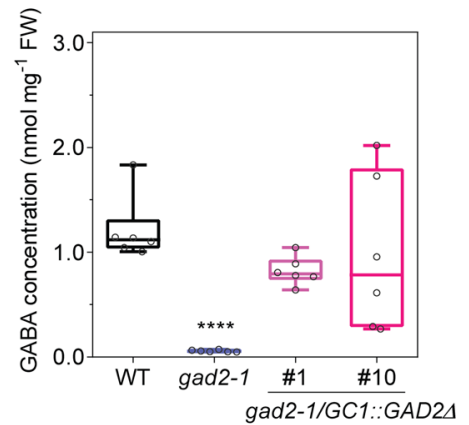

e

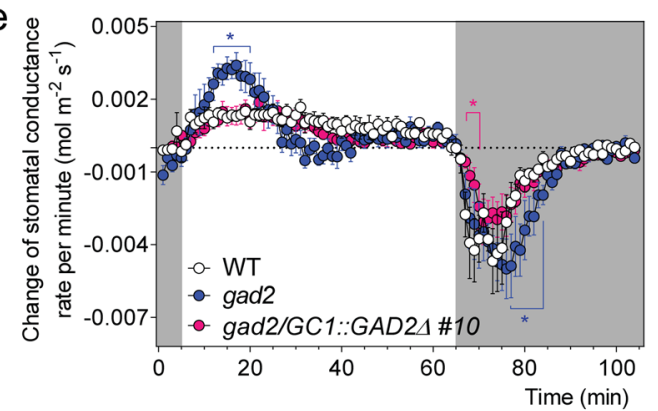

C

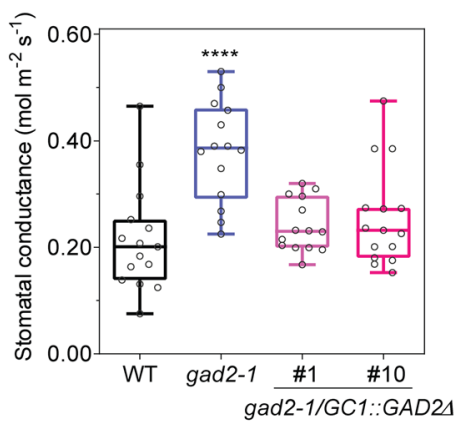

$f$

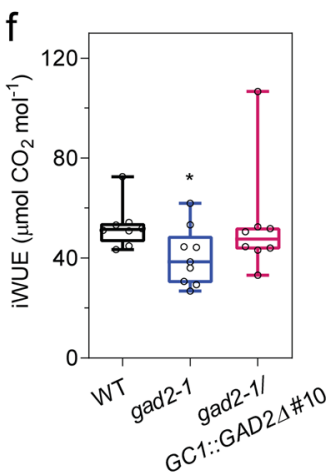

h

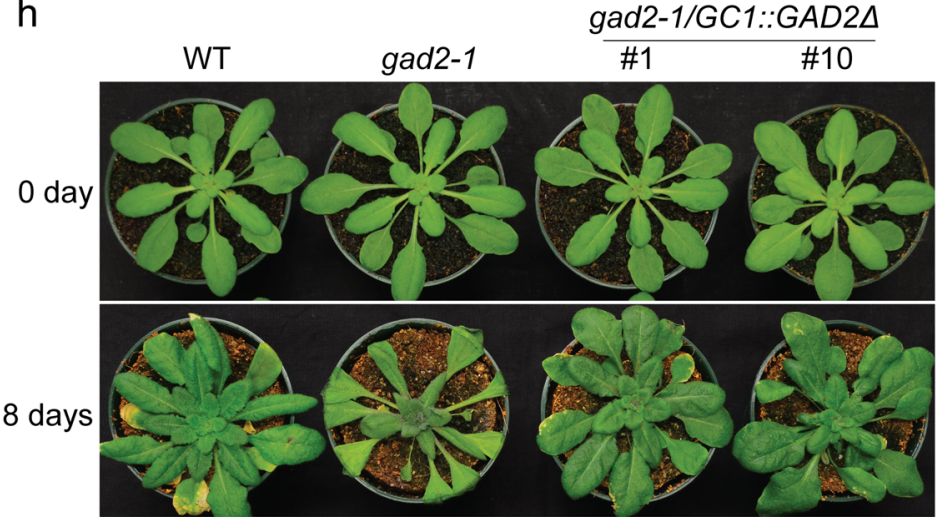

Fig. 4 Guard cell GABA regulates water loss and drought tolerance. a Representative confocal images of gad2-1 plants expressing GC1::GAD2L-GFP (gad2-1/GC1::GAD2D-GFP); GFP fluorescence and chlorophyll autofluorescence (blue) of the leaf abaxial side of 3-4-week-old gad2-1/GC1::GAD24-GFP plant indicates that the GC1 promoter drives GAD2 2 expression specifically in guard cells, similar pattern images are obtained from multiple gad2-1/GC1:: GAD2 - -GFP plants, scale bars $=50 \mu \mathrm{m}$. b Leaf GABA accumulation of 5-6-week-old A. thaliana WT, gad2-1, gad2-1/GC1::GAD2 \#1 and \#10 plants grown under control conditions, $n=6$. c Stomatal conductance of WT $(n=15)$, gad2-1 $(n=14)$, gad2-1/GC1::GAD2 $\# 1(n=14)$ and \#10 ( $n=15)$ plants under control conditions determined using an AP4 porometer, data collected from two independent batches of plants. $\mathbf{d}$ Stomatal conductance of WT, gad2-1 and gad2-1/GC1::GAD2 \#10 plants in response to dark (shaded region) and $150 \mu \mathrm{mol} \mathrm{m}-2 \mathrm{~s}^{-1}$ light (white region), measured using a LI-COR LI-6400XT. e Change in stomatal conductance each minute calculated using dConductance/dt ( $\mathrm{min}$ ) of the data represented in d. f iWUE of WT, gad2-1 and gad2-1/ GC1::GAD2 $\Delta$ plants was calculated based on the ratio of photosynthetic rate (Supplementary Fig. $8 \mathrm{~h}$ ) versus stomatal conductance represented in $\mathbf{d} ; n=8$ individual plants for WT, $n=9$ for gad2-1 and $n=8$ for gad2-1/GC1::GAD2 $\# 10$, data collected from two independent batches of plants (d-f). $\mathbf{g}$ Relative leaf water content of WT, gad2-1, gad2-1/GC1::GAD2 \#1 and \#10 plants following drought treatment for $0,1,3,5$ and 7 days; $n=4$ for $0,1,3$ and 5 days samples and $n=5$ for 7 days samples, except that $n=3$ for 0-day gad2-1 and 1-day gad2-1/GC1::GAD2 \#1. h Representative images of WT, gad2-1, gad2-1/ GC1::GAD2 \#1 and \#10 plants (shown in $\mathbf{i}$ ) before ( 0 day) and after ( 8 days) drought treatment as indicated. All data are plotted with box and whiskers plots: whiskers plot represents minimum and maximum values, and box plot represents second quartile, median and third quartile (b, $\mathbf{c}, \mathbf{f})$, or data are represented as mean \pm s.e.m $(\mathbf{d}, \mathbf{e}, \mathbf{g})$; statistical difference was determined using by two-sided Student's $t$ test (f), one-way ANOVA (b, c) or two-way ANOVA $(\mathbf{e}, \mathbf{g}) ;{ }^{\star} P<0.05$ and ${ }^{\star \star \star \star} P<0.0001$.

GABA signalling regulating WUE and drought resilience is ALMT9 dependent. ALMTs are plant-specific anion channels that share no homology to Cys-loop receptors except a region of 12 amino acid residues predicted to bind $\mathrm{GABA}$ in $\mathrm{GABA}_{\mathrm{A}}$ receptors ${ }^{14,18}$. In animals, ionotropic GABA receptors are stimulated by GABA; in contrast, anion currents through ALMTs are inhibited by $\mathrm{GABA}^{10,11}$. There are a number of ALMTs expressed in guard cells that contain the putative GABA binding motif and have the potential to transduce the GABA signal, with most having been shown to have a role in regulating stomatal movement ${ }^{20-22,40}$. For instance, ALMT12 (also called QUAC1, quickly-activation anion conductance 1) is a plasma membrane localised anion channel, which moves anions out of the guard cell during guard cell closure ${ }^{20}$.

Under the conditions tested here, the impact of GABA on stomatal closure appears to be limited to epidermal peels, it is not 

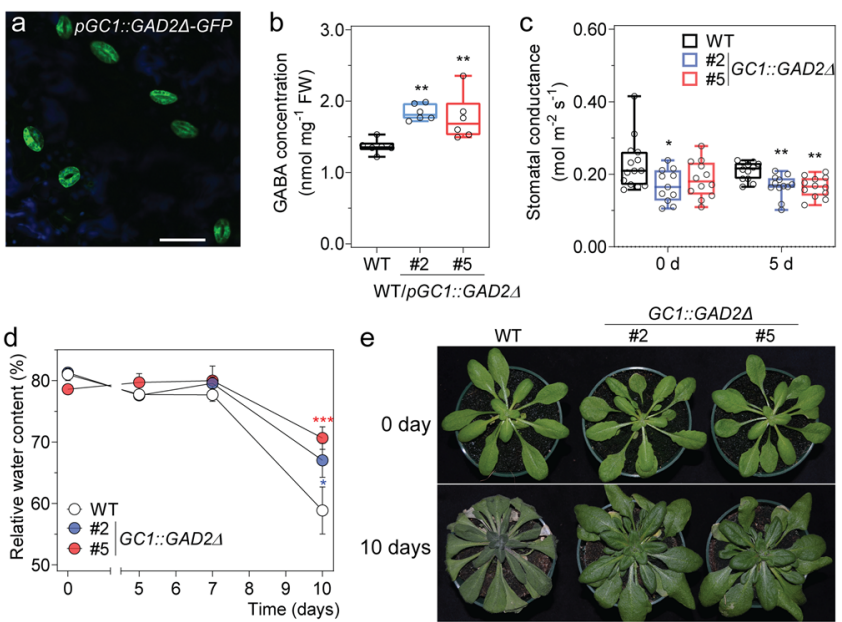

Fig. 5 Guard cell overexpression of GAD2 $\triangle$ decreases plant water loss and increases drought survival. a Representative confocal images of $A$. thaliana wild-type plants expressing GC1::GAD2L-GFP; GFP fluorescence and chlorophyll autofluorescence (blue) of the leaf abaxial side of 3-4-week-old plants, similar pattern images are obtained from multiple wild-type plants expressing GC1::GAD2 $\Delta$-GFP plants, scale bars $=50 \mu \mathrm{m}$. b GABA accumulation in the leaves of 5-6-week-old Arabidopsis wild type, GC1:: GAD2 $\# 2$ and \#5 plants; $n=6$. c Stomatal conductance of WT, wild-type Arabidopsis expressing GAD2A in the guard cells using the GC1 promoterGC1::GAD2 $\# 2$ and \#5 plants before (0 day) and after (5 days) drought treatment determined using an AP4 porometer; $n=14$ for $\mathrm{WT}, n=11$ for GC1::GAD2 $\# 2$ and $n=15$ for GC1::GAD2 $\# 2$ at 0 day and $n=12$ for WT, GC1::GAD2 $\# 2$ and \#5 at 5 days. d Relative leaf water content of WT, GC1:: GAD2 $\# 2$ and \#5 plants following drought treatment for 0, 5, 7 and 10 days; $n=6$ for 0 and 5 days all samples, except $n=18$ for WT at 10 days, $n=12$ for GC1::GAD2 $\# 2$ at 10 days and $n=13$ for GC1::GAD2 $\# 5$ at 10 days. e Representative images of WT, GC1::GAD2 \#2 and \#5 plants before ( 0 day) and after ( 10 days) drought treatment as indicated. Pot size 2.5 inch diameter $\times 2.25$ inch height (LI-COR). All data are plotted with box and whiskers plots: whiskers plot represents minimum and maximum values, and box plot represents second quartile, median and third quartile (b, c), or data are represented as mean \pm s.e.m (d); statistical difference was determined using one-way ANOVA $(\mathbf{b}, \mathbf{c})$ or two-way ANOVA (d); ${ }^{*} P$ $<0.05,{ }^{* \star} P<0.01$ and ${ }^{* \star *} P<0.001$.

seen in intact leaves. However, epidermal peels still represent an assay system that can be used to test whether ALMT might transduce the inhibitory effect of GABA on closure. We observed that, unlike wild-type plants, stomatal closure in almt12 knockouts was insensitive to GABA or muscimol when transitioning from light-to-dark (Supplementary Fig. 13a). In contrast, stomatal opening of almt12 lines showed wild-type-like sensitivity to GABA or muscimol when transitioning from dark to light (Supplementary Fig. 13b). These data indicate that ALMT12 is a plasma membrane GABA target that affects stomatal closure in response to dark-in epidermal peels at least.

However, if GABA inhibition of ALMT12/QUAC1 played a significant role during drought, then the resulting inhibition of closure would translate into an increase in water loss compared to wild-type plants during closure. As we found no evidence that GABA had an effect on closure in intact leaves, under a light-todark transition as measured by stomatal conductance or transpiration (Fig. 2c; Supplementary Fig. 2a), and the fact that GABA accumulation led to a net decrease in water loss and improvement in drought resilience, ALMT12 is unlikely to be a major target contributing to this outcome. We therefore focused on tonoplast-localised ALMTs that are involved in stomatal pore opening $^{21}$, as this is the process where GABA has its predominant affect in intact leaves.

ALMT9 is the major tonoplast-localised channel involved in anion uptake into guard cell vacuoles during stomatal opening, but has no documented role in closure ${ }^{21}$. We hypothesised that GABA might target and inhibit ALMT9 activity to reduce the rate or extent of stomatal opening. We initially attempted in vitro electophysiological studies to examine the impact of GABA on ALMT9-induced currents, but were unable to consistently detect stable currents following heterologous expression in either Xenopus laevis oocytes or tobacco mesophyll cells ${ }^{21,41}$. Therefore, we examined the potential regulation of ALMT9 by GABA by focusing solely on in planta studies as it is difficult to faithfully replicate regulatory pathways from guard cells in heterologous systems, e.g. ${ }^{42-47}$. In the first instance, we independently crossed two almt9 alleles (almt9-1 and almt9-2) with gad2-1. We found that, similar to gad2, both double mutants (gad2-1/almt9-1 and gad2-1/almt9-2) maintained low GABA accumulation in their leaves (Fig. 6a, b; Supplementary Fig. 14a, e). However, both gad2-1/almt9-1 and gad2-1/almt9-2 had wild-type-like stomatal conductance and aperture unlike gad2-1 where both these parameters are high (Fig. 6c, d; Supplementary Fig. 14d, f). Furthermore, guard cell-specific complementation of gad2-1/ almt9-1 by GAD2A did not alter stomatal conductance (Supplementary Fig. 14a-d). Collectively, these data are consistent with ALMT9 being required for GABA to regulate gas exchange via stomatal control. An interesting additional observation was that the loss of ALMT9 in gad2-1 also resulted in ABA inducing stomatal pore closure to wild-type levels (Supplementary Fig. 14g-j), indicating that, although ALMT9 is a channel that regulates stomatal opening, it can influence the extent to which stomatal pores close under certain conditions (in epidermal peels at least). The incomplete stomatal closure of $\mathrm{gad} 2$ coupled to its greater stomatal opening may further contribute to its drought sensitivity. These findings are consistent with the regulation of stomatal aperture being a dynamic equilibrium between the pathways that regulate stomatal opening and closure, with stomatal aperture being weighted towards a particular state dependent upon the dominant stimuli ${ }^{48,49}$.

To further test whether ALMT9 transduces GABA signalling, we examined the effect of GABA on regulating stomatal opening in almt 9 mutant plants. In wild-type plants, we previously showed that light-induced stomatal opening was inhibited by exogenous GABA (Fig. 2a) or muscimol (Supplementary Fig. 1a). In almt9 lines, exogenous GABA or muscimol did not antagonise stomatal opening (Fig. 7a, b; Supplementary Fig. 15a, b), whereas darkinduced stomatal closure in almt9 retained its GABA sensitivity (Fig. 7c, d; Supplementary Fig. 15c, d). These results are consistent with GABA reducing stomatal opening via negative regulation ALMT9-mediated $\mathrm{Cl}^{-}$uptake into guard cell vacuoles. Furthermore, it strongly indicates the corollary of this finding, that the higher stomatal conductance phenotype of gad2 is the result of greater ALMT9 activity due to its lack of inhibition by GABA.

We tested this hypothesis by attempting to complement almt 9 plants with either the native channel or a site-directed ALMT9 mutant (ALMT9 ${ }^{\mathrm{F} 243 \mathrm{C} / \mathrm{Y} 245 \mathrm{C} \text { ). The mutations within ALMT9 }}{ }^{\mathrm{F} 243 \mathrm{C} /}$ $\mathrm{Y} 245 \mathrm{C}$ are in the 12 amino acid residue motif that shares homology with a GABA binding region in mammalian $\mathrm{GABA}_{\mathrm{A}}$ receptors ${ }^{14,18}$. Mutations in the aromatic amino acid residues in this motif have been shown for other ALMTs to result in active channels that are not inhibited by GABA when tested in heterologous systems ${ }^{36,39}$ (Fig. 8; Fig. 9). However, no in planta tests have been conducted to date-for any ALMT - to determine whether mutations in this region result in a transport competent protein that lacks GABA sensitivity. Here, we observed that ALMT9 and ALMT9F243C/Y245C had similar 
a
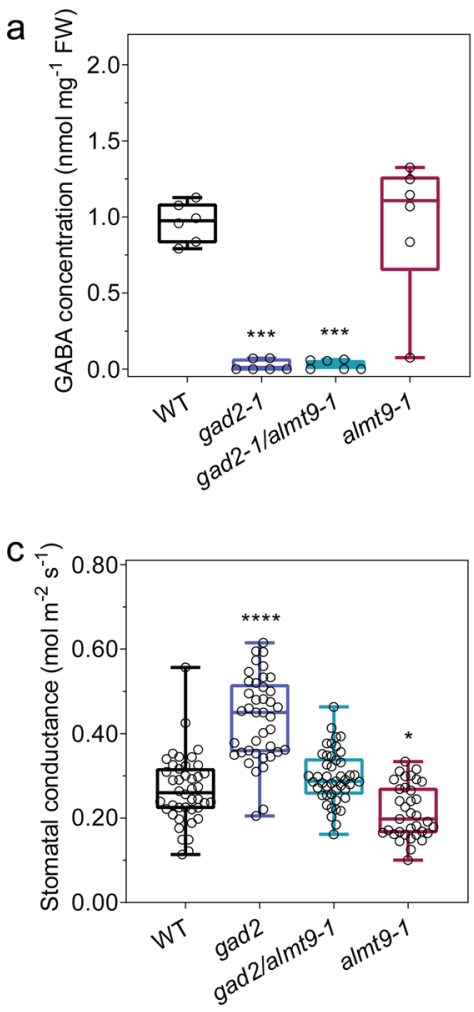

b $\widehat{s}$
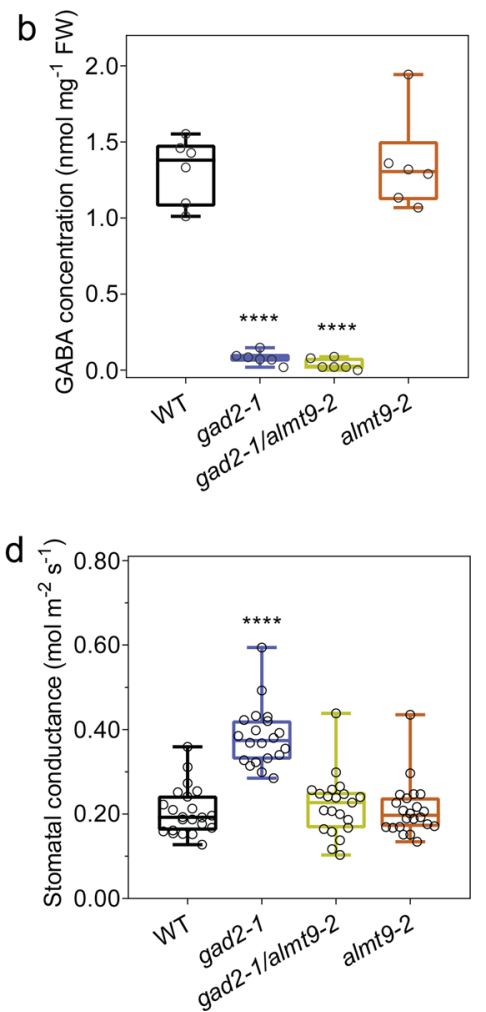

Fig. 6 The loss of ALMT9 suppresses the gad2 mutant stomatal phenotype. a-d Leaf GABA concentration (a, b) and stomatal conductance (c, d) of 5-6week-old A. thaliana WT, gad2-1, gad2-1/almt9-1, almt9-1, gad2-1/almt9-2 and almt9-2 plants; $n=6$ plants (a, b); $n=42$ for WT, $n=40$ for gad2-1, $n=45$ for gad2-1/almt9-1 and $n=35$ for almt9-1, data collected from four independent batches of plants (c); $n=22$ for WT, $n=20$ for gad2-1, $n=21$ for gad2-1/ almt9-2 and $n=22$ for almt9-2, data collected from two independent batches of plants (d); data (a, c) were extracted respectively from Supplementary Fig. 13b, c. All data are plotted with box and whiskers plots: whiskers plot represents minimum and maximum values, and box plot represents second quartile, median and third quartile; statistical difference was determined by one-way ANOVA, ${ }^{\star} P<0.05,{ }^{\star \star \star} P<0.001$ and ${ }^{\star \star \star \star} P<0.0001$.
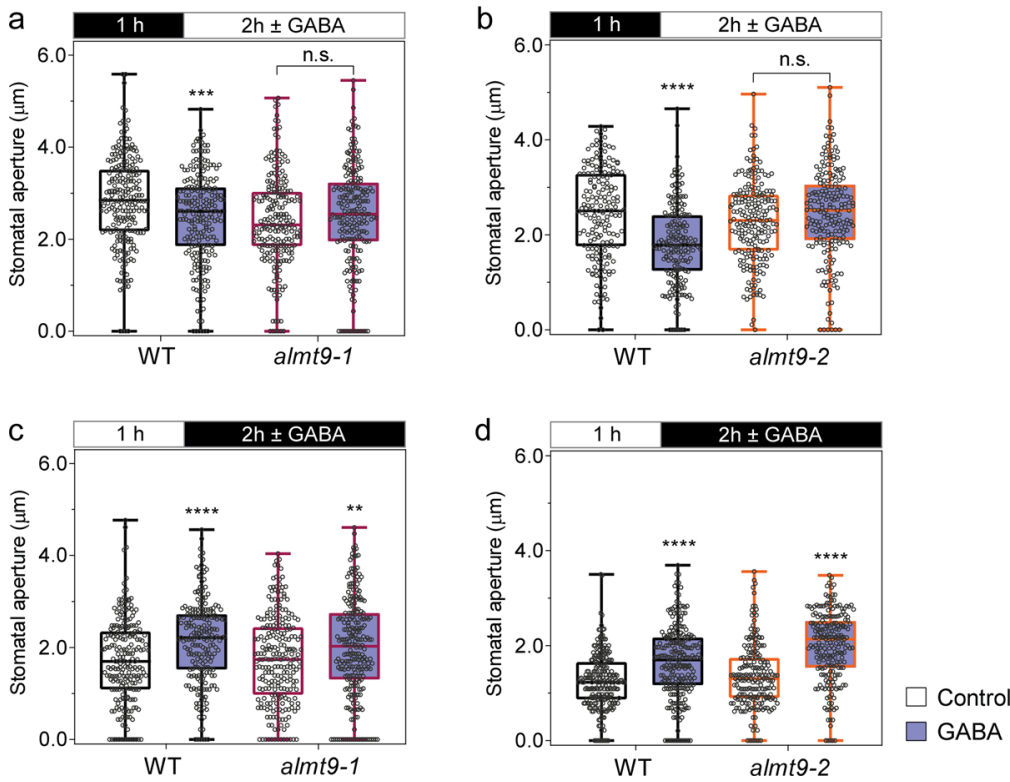

Fig. 7 The loss of ALMT9 abolishes GABA inhibition of stomatal opening but does not affect closure. a-d Arabidopsis WT and almt9 knockout plant stomatal aperture in response to light or dark. Epidermal strips were pre-incubated in stomatal measurement buffer for $1 \mathrm{~h}$ under dark (a, b) or light (c, d), followed by $2 \mathrm{~h}$ in light $(\mathbf{a}, \mathbf{b})$ or dark (c, d) as indicated by black (dark) or white (light) bars above graphs with $\pm 2 \mathrm{mM} \mathrm{GABA} ; n=236$ for WT and $n=221$ for almt9-1 with control treatment, $n=229$ for WT and $n=215$ for almt9-1 with GABA treatment (a); $n=223$ for WT and $n=242$ for almt9-1 with control treatment, $n=215$ for WT and $n=256$ for almt9-1 with GABA treatment (b); $n=183$ for WT and $n=189$ for almt9-2 with control treatment, $n=210$ for WT and $n=197$ for almt9-2 with GABA treatment (c); $n=236$ for WT and $n=243$ for almt9-2 with control treatment, $n=202$ for WT and $n=220$ for almt9-2 with GABA treatment (d). All data are plotted with box and whiskers plots: whiskers plot represents minimum and maximum values, and box plot represents second quartile, median and third quartile; statistical difference was determined by two-way ANOVA, ${ }^{\star \star} P<0.01,{ }^{\star \star \star} P<0.001$ and ${ }^{\star \star \star \star} P<0.0001$. 

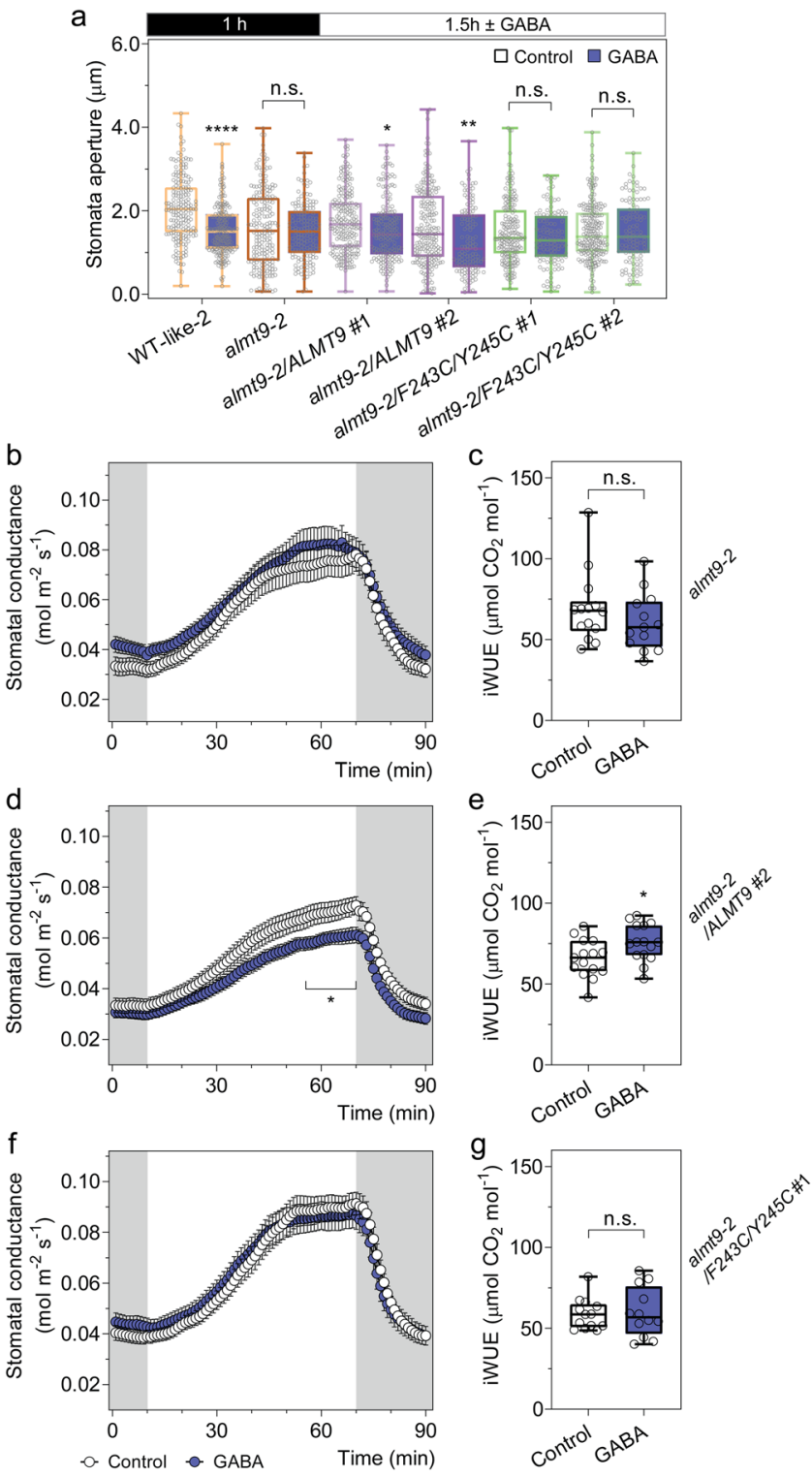

expression in almt9-2 complementation lines and the mutations (in ALMT9F243C/Y245C) did not alter the membrane localisation with both versions of the ALMT9 protein being clearly present on the tonoplast (Supplementary Fig. 16). Further, we found that similar to almt9 lines, almt9-2 expressing ALMT9F243C/Y245C was insensitive to GABA during a dark-to-light transition assayed on epidermal peels and detached leaves, for stomatal opening and stomatal conductance, respectively; this contrasts the GABA sensitivity of wild-type plants and plants expressing native ALMT9 in the almt9-2 background (Figs. 2c and 8a, b, d, f; Supplementary Fig. 17a, b, d, e, g, h). Furthermore, instantaneous iWUE/WUE of almt9-2 was improved by native ALMT9 complementation, but not ALMT9F243C/Y245C (Fig. 8c, e, g; Supplementary Fig. 17c, f, i). Steady-state stomatal conductance and aperture of $A L M T 9^{F 243 C / Y 245 C}$ lines were also significantly greater than that of wild-type and almt 9 lines and were insignificant from gad2-1 under standard conditions (Fig. 9; Supplementary Fig. 17j). This result indicates that we successfully complemented almt 9 with an active, but GABA-insensitive form of ALMT9, and that this increased transpirational water loss over wild-
Fig. 8 ALMT9 but not ALMT9F243C/Y245C restores the GABA sensitivity of almt9-2. a Stomatal aperture measurement of A. thaliana WT, almt9-2 and complementation lines. Epidermal strips were pre-incubated in stomatal measurement buffer for $1 \mathrm{~h}$ under dark, followed by a $1.5 \mathrm{~h}$ dark-tolight transition, as indicated above graphs by black (dark) or white (light) bars, $\pm 2 \mathrm{mM} \mathrm{GABA} ; n=189$ (control) and $n=195$ (GABA) for WT-like 2 (segregated from almt9-2)21, $n=197$ (control) and $n=153$ (GABA) for almt9-2, $n=213$ (control) and $n=178$ (GABA) for almt9-2 complement with 35S::ALMT9 \#1 (almt9-2/ALMT9 \#1), $n=219$ (control) and $n=127$ (GABA) for almt9-2/ALMT9 \#2, $n=195$ (control) and $n=115$ (GABA) for almt9-2 complemented with 35S::ALMT9 with double mutation F243C/

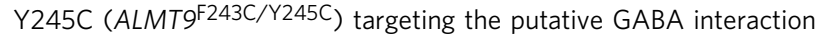
residues $^{18,36,39}$ (almt9-2/F243C/Y245C \#1), $n=221$ (control) and $n=109$ (GABA) for almt9-2/F243C/Y245C \#2 with control treatment. b-g Leaf feeding assay of almt9-2 and complementation lines. Stomatal conductance of detached leaves from 5-6-week-old Arabidopsis almt9-2, almt9-2/ALMT9 \#2 and almt9-2/F243C/Y245C \#1 plants was recorded using a LI-COR LI$6400 X \mathrm{~T}$ in response to dark (shaded region) and $200 \mu \mathrm{mol} \mathrm{m} \mathrm{m}^{-2} \mathrm{~s}^{-1}$ light (white region), fed with artificial xylem sap solutions $\pm 4 \mathrm{mM} \mathrm{GABA}$ (b, d, f). The iWUE of almt9-2 (c), almt9-2/ALMT9 \#2 (e) and almt9-2/F243C/ Y245C \#1 ( $g$ ) detached leaves was calculated based on the ratio of photosynthetic rate (Supplementary Fig. 17b, e, h) versus stomatal conductance (b, d, f); $n=14$ (control) and $n=13$ (GABA) for almt9-2 (b, c); $n=15$ (control and GABA) for almt9-2/ALMT9 \#2 (d, e); $n=13$ (control) and $n=12$ (GABA) for almt9-2/F243C/Y245C \#1 (f, g). All data are plotted with box and whiskers plots: whiskers plot represents minimum and maximum values, and box plot represents second quartile, median and third quartile $(\mathbf{a}, \mathbf{c}, \mathbf{e}, \mathbf{g})$, or data are represented as mean \pm s.e.m $(\mathbf{b}, \mathbf{d}, \mathbf{f})$ statistical difference was determined by two-sided Student's $t$ test; ${ }^{\star} P<$ $0.05,{ }^{\star \star} P<0.01,{ }^{\star \star \star \star} P<0.0001(\mathbf{a}-\mathbf{g})$.

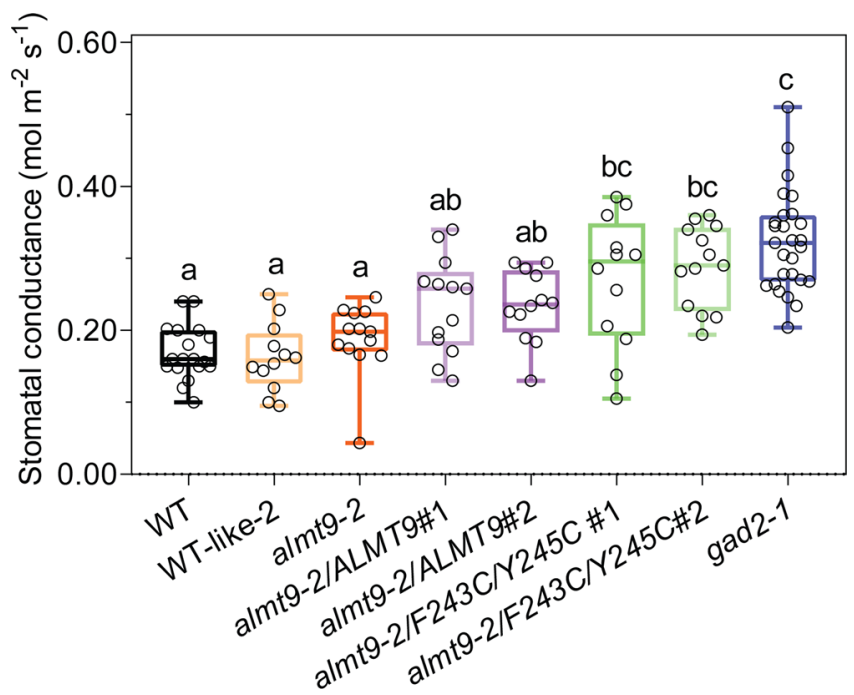

Fig. 9 ALMT9F243C/Y245C increases steady-state stomatal conductance. Stomatal conductance of 5-6-week-old Arabidopsis WT, gad2-1, almt9-2 and complementation lines determined using an AP4 Porometer; $n=18$ for WT, $n=12$ for WT-like 2, almt9-2/ALMT9 \#2 and almt9-2/F243C/Y245C $\# 1, n=13$ for almt9-2, almt9-2/ALMT9 \#1 and almt9-2/F243C/Y245C \#2, $n=27$ for gad2-1. All data are plotted with box and whiskers plots: whiskers plot represents minimum and maximum values, and box plot represents second quartile, median and third quartile; statistical difference was determined by one-way ANOVA, the letters $a, b$ and $c$ represent data groups that are not statistically different, $P<0.05$. 
type levels. These data are completely consistent with ALMT9 being a GABA target that regulates plant water loss, even under nonstressed conditions, through modulation of ALMT9 activity. The GABA effect is then amplified under a water deficit when GABA concentration increases. We propose that GABA accumulation has a role in promoting drought resilience by reducing the amplitude of stomatal re-opening each morning, which minimises whole plant water loss. As such, the GABA-ALMT pathway is a strong candidate for constituting the ABA-independent stress memory of a decreased soil water status that has been previously proposed without mechanistic attribution ${ }^{50,51}$.

\section{Discussion}

The data in this manuscript have unveiled a GABA signalling pathway in plants, which can be summarised by the simplified models presented in Fig. 10. We propose that cytosolic GABA signals, generated by GAD2, modulate stomatal opening, WUE and drought resilience transduced through negative regulation of ALMT9 activity (Fig. 10).

Collectively our use of leaf feeding, knockouts, complementation and point mutagenesis strongly suggests ALMT9 is an essential and major component transducing GABA signalling in guard cells during well-watered and drought conditions. As has become evident for other guard cell based signalling pathways through their examination over time ${ }^{42-47}$, we are cognizant of the potential that other GABA response elements, including other ALMT, may be involved in transducing and fine-tuning this signalling pathway. Our finding that GABA does not impact stomatal closure in epidermal peels of almt12 knockouts infers a potential role for this plasma membrane localised ALMT12 in transducing guard cell GABA signals. The fact that light-induced stomatal opening and darkinduced stomatal closure was completely GABA insensitive in almt9xalmt12 knockouts (Supplementary Fig. 18) suggests that both channels have the potential to transduce the major effects of GABA in guard cells.

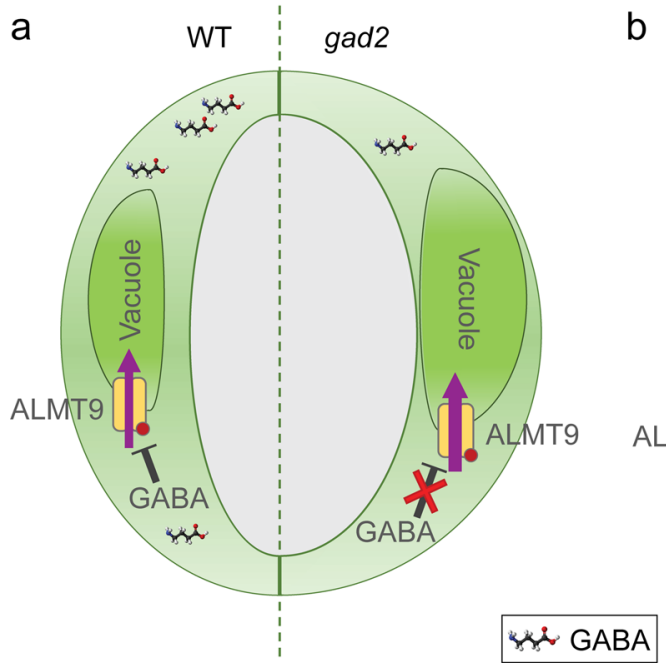

However, it is interesting that GABA inhibition of stomatal opening was consistently seen between epidermal peel assays and leaf feeding, whilst GABA only inhibited stomatal closure during isolated epidermal peel experiments, but not when it was fed to leaves. This suggests that GABA acts through ALMT12 on processes associated with stomatal closure, but in the context of an intact leaf this phenotype is lost, which is likely due to the loss of functional epidermal and/or mesophyll cells. This is consistent with the growing body of evidence that indicates stomatal aperture experiments on isolated epidermal peels require validation via studies on intact leaves to avoid overinterpreting potential artifacts from this reductionist system. However, it also means we cannot fully rule out whether GABA inhibition of stomatal closure does have a role under certain physiological scenarios that are yet to be identified. Therefore, in future studies, it would be pertinent to examine whether ALMT12-dependent GABA inhibition of stomatal closure has a physiological role in transducing GABA signals in conditions not examined here, and, more broadly, whether other ALMTs or additional elements are involved in GABA signal transduction.

ALMT activity appears to be regulated by a suite of factors including anions, $\left(\mathrm{Al}^{3+}\right.$ for ALMT1), $\mathrm{pH}$, ATP, voltage and $\mathrm{GABA}^{52}$. As such, it is becoming clear that ALMTs have the potential to act as a key signalling hub in a variety of physiological processes. Following on from this study, leading on from the observed GABA modulation of $\mathrm{ABA}, \mathrm{H}_{2} \mathrm{O}_{2}$ and coronatine effects on stomata, the investigation into cross-talk between GABA and other signals for ALMT9, in particular, and ALMTs, in general, provides the basis for future research areas. Such studies will be able to resolve questions such as 'whether GABA can act directly on guard cell ALMTs?', as appears to occur for wheat ALMT1 18,39 , or 'whether other signalling intermediates are also involved?'. GABA inhibition of the wheat ALMT1 anion conductance was recently found to occur from the cytosol only, by reducing the open probability of the channel to anions ${ }^{39}$. However, that study was unable to determine whether this occurred through permeation of uncharged GABA through the ALMT

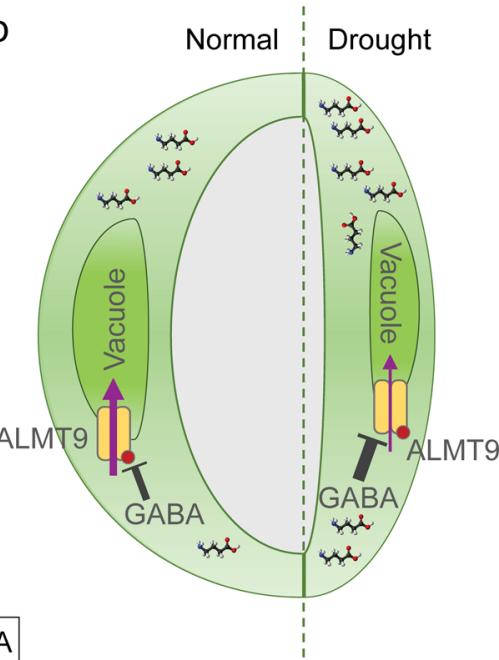

Fig. 10 Proposed model of GABA-mediated signalling for the regulation of water use efficiency. a Cytosolic guard cell GABA negatively regulates ALMT9-mediated anion uptake into guard cell vacuoles, which fine tunes stomatal opening (left guard cell of pair). Depletion of GABA accumulation in the leaves of GAD2 loss-of-function mutant (gad2) de-regulates ALMT9, maximizing anion uptake and accumulation in guard cell vacuoles. This leads to a more open stomatal pore, greater water loss and lower WUE of plants (right guard cell of pair). This stomatal phenotype can be replicated by replacing F243/Y245 (red dot) with two cysteines, which abolishes GABA sensitivity of ALMT9. b Leaf GABA synthesized and accumulated during water deficit reduces ALMT9-mediated vacuolar anion uptake into guard cells, which requires amino acid residues F243/Y245 (red dot) (right guard cell of pair). This reduces stomatal opening, reducing the pore aperture and enhances plant WUE under drought stress compared to guard cells under standard conditions in the light (left guard cell of pair). Note: We have excluded ALMT12 from this model as we did not find a role for this protein in GABA modulation of water use efficiency in planta, despite its role in GABA modulation of stomatal aperture found within epidermal peels. 
pore or through GABA binding modifying channel structure ${ }^{39}$. Cytosolic GABA inhibition was dependent upon the putative GABA binding residue F213 (equivalent to F243 in ALMT9, which is also predicted to face the cytosol) ${ }^{39,53}$. Our study therefore highlights the real need to definitively determine whether GABA binds to ALMTs or whether the identified amino acid residues affect GABA sensitivity independent of anion permeability through other means. For instance, future studies should address whether GABA permeability of ALMTs has a role in signal transduction in guard cells and the regulation of other physiological processes ${ }^{36}$. These later questions would be aided by the determination of GABA concentrations in different cell types and compartments to further understand the co-ordination of GABA signalling across membranes, leaves and other organs, and this could be achieved through the deployment of novel GABA sensors, as recently used in animal tissues ${ }^{54,55}$.

GABA concentration oscillates over diel cycles and increases in response to multiple abiotic and biotic stresses including drought, heat, cold, anoxia, wounding pathogen infection and salinity ${ }^{13}$. ALMTs have been implicated in modulating multiple developmental and physiological processes in plants ${ }^{20-22,56-58}$ including those underpinning nutrient uptake and fertilization that are affected by GABA ${ }^{18,59,60}$. Therefore, the discovery that GABA regulates ALMT to form a physiologically relevant signalling mechanism in guard cells is likely to have broad significance beyond stomata, particularly during plant responses to environmental transitions and stress.

GABA's effect on stomata appears to be conserved across a large range of crops from diverse clades including important monocot and dicot crops (Supplementary Fig. 5), indicating that GABA may well be a stomatal signal of economic significance. As we find that the genetic manipulation of cell-type specific GABA metabolism can reduce water loss leading to improved drought performance, our work opens up alternative ways for manipulating crop stress resilience. This statement is tempered in the knowledge that GABA modulated stomatal signalling in the face of another signal and did not stimulate changes in stomatal aperture itself. GABA's role appears to be that of fine-tuning stomatal aperture. Our data suggest that GABA modulated stomatal movement occurs in response to light and dark and low concentrations of signal intermediates, but in the face of a strong stress stimulus its affects may be overridden. As such, GABA may well provide a direct link between the metabolic status of the cell - GABA being produced in the cytosol in times of stress as a bypass of several reactions of the TCA cycle-to regulate and sustain a certain physiological process prior to it being shut down via a more severe stress response pathway. More broadly, this study also provides proof that GABA is a plant signalling molecule and not just a plant metabolite ${ }^{12,16}$, and in so doing, we conclude that GABA is an endogenous signalling molecule beyond the animal and bacterial kingdoms, enacted through distinct and organism specific mechanisms.

\section{Methods}

Plant materials and growth conditions. All experiments were performed on $A$. thaliana were in the Columbia-0 (Col-0) ecotype background, unless stated. Arabidopsis wild type, T-DNA insertion mutant and other transgenic plants were germinated and grown on $1 / 2$ Murashige and Skoog (MS) medium with $0.8 \%$ phytagel for 10 days before being transferred to soil for growth in short-day conditions (100-120 $\mu \mathrm{mol} \mathrm{m}^{-2} \mathrm{~s}^{-1}, 10 \mathrm{~h}$ light/14 h dark) at $22^{\circ} \mathrm{C}$. The T-DNA insertion mutant gad2-1 (GABI_474_E05) and gad2-2 (SALK_028819) were obtained from the Arabidopsis Biological Resource Centre (ABRC). gad2-1 was selected using primer sets:

gad2_LP1 (5'-TATCACGCTAACACCTAACGC-3'), gad2_RP1 (5' TTCAAGGTTTGTCGGTATTGG-3') and GABI_LB (5'-GGGCTACAC TGAATTGGTAGCTC- $\left.3^{\prime}\right)$ for removing the second T-DNA insert; gad2_LP2 (5' ACGTGATGGATCCAGACAAAG-3'), gad2_RP2 (5'-TCTTCATTTCCAC ACAAAGGC-3') and GABI_LB for isolation of the GAD2 (At1g65960) T-DNA insertion. gad2-2 was selected using primer sets: gad2-2_LP (5'-AGTTGTATGAA AGTTCATGTGGC-3'), gad2-2 RP (5'-TCGACCACGAGATTTTAATGG -3') and SALK_LB (5'-ATTTTGCCGATTTCGGAAC-3'). almt9-1 (SALK_055490), almt9-2 (WiscDsLox499H09)), almt12-1 (SM_3_38592) and almt12-2 (SM_3_1713) were selected as described previously ${ }^{20,21}$. The double mutant lines gad2-1/almt9-1, gad2-1/almt9-2, almt9-2/12-1 and almt9-2/12-2 were obtained, respectively, from crossing the respective mutants. The mesophyll enhancer-trap line JR11-2 in the Col-0 background was kindly provided by K. Baerenfaller (ETH Zurich $)^{61}$. JR11-2 (Col-0) and gad2-1/JR11-2 were segregated from crossing gad2-1 with JR11-2. JR11-2 was selected using primer sets: JR11-2_LP (5'-TTATTTAGGG AAATTACAAGTTGC-3'), JR11-2_RP (5'-AGACACATTTAATAACATTACAAC AAA-3 $\left.3^{\prime}\right)$ and JR11-2_LB (5'-GTTGTCTAAGCGTCAATTTGTTT-3 $\left.{ }^{\prime}\right)^{62}$. All experiments were performed on stable $\mathrm{T}_{3}$ transgenic plants or confirmed homozygous mutant lines. The other plants $V$. faba, N. benthamiana and G. max were grown in soil in long-day conditions $\left(400 \mu \mathrm{mol} \mathrm{m}{ }^{-2} \mathrm{~s}^{-1}, 16 \mathrm{~h}\right.$ light $/ 8 \mathrm{~h}$ dark, $28^{\circ} \mathrm{C} / 25^{\circ} \mathrm{C}$ ). H. vulgare (barley) cv. Barke was grown in a hydroponic system with half-strength Hoagland's solution in long-day conditions $\left(150 \mu \mathrm{mol} \mathrm{m}^{-2} \mathrm{~s}^{-1}, 16 \mathrm{~h}\right.$ light $/ 8 \mathrm{~h}$ dark, $\left.23^{\circ} \mathrm{C}\right)^{63}$

Gene cloning and plasmid construction. For guard cell-specific complementation, the constitutively active form of GAD2 with a truncation of the calmodulin binding domain $(G A D 2 \triangle)^{31,33}$ and the full-length GAD2 coding sequence (GAD2) was driven by a guard cell-specific promoter GC1 $(-1140 /+23)^{32}$, as designated $G C 1:: G A D 2 \triangle$ and $G C 1:: G A D 2$, respectively. PCR reactions first amplified the truncated $G A D 2 \triangle$ with a stop codon and $G C 1$ promoter $(G C 1)$ separately using Phusion ${ }^{\boxplus}$ High-Fidelity DNA Polymerase (New England Biolabs) with the primer sets: GAD2_forward (5'-CACTACTCAAGAAATATGGTTTTGACAAAAACC GC- $\left.3^{\prime}\right)$ and GAD2_truncated_reverse (5'-TTATACATTTTCCGCGATCCC-3' $)$; GC1_forward (5'-CACCATGGTTGCAACAGAGAGGATG-3') and GC1_reverse (5'-ATTTCTTGAGTAGTGATTTTGAAG-3'). This was followed by an overlap PCR to fuse the GC1 promoter to GAD2 $(G C 1:: G A D 2 \Delta)$ with the GC1_forward and GAD2_truncated_reverse primer set. The same strategy was used to amplify GC1::GAD2 2 without a stop codon (GC1::GAD2A-stop), GC1::GAD2 and GC1:: GAD2 without a stop codon (GC1::GAD2-stop) with different primer sets: (1) GC1: GAD2 $\triangle$-stop amplified with GAD2_forward and GAD2_truncated-stop_reverse (5'-TACATTTTCCGCGATCCCT-3'); (2) GC1::GAD2 amplified with GAD2_forward and GAD2_reverse (5'-TTAGCACACACCATTCATCTTCTT-3') and (3) GC1::GAD2-stop amplified with GAD2_forward and GAD2-stop_reverse ( $5^{\prime}$ CACACCATTCATCTTCTTCC-3'). The fused PCR products were cloned into the pENTR/D-TOPO vector via directional cloning (Invitrogen). pENTR/D-TOPO vectors containing $G C 1:: G A D 2 \triangle$ or $G C 1:: G A D 2$ were recombined into a binary vector pMDC $99^{64}$ by an LR reaction using LR Clonase II Enzyme mix (Invitrogen) for guard cell-specific complementation, after an insertion of a NOS Terminator into this vector. A pMDC99 vector was cut by $\mathrm{PacI}$ (New England Biolabs) and ligated with NOS terminator flanked with PacI site using T4 DNA ligase (New England Biolabs). This NOS terminator flanked with PacI site was amplified with primer set: nos_PacI_forward (5'-TACGTTAATTAAGAATTTCCCCGAT- $3^{\prime}$ ) and nos_PacI_reverse (5'-GCATTTAATTAAAGTAACATAGATGACACC-3 $\left.3^{\prime}\right)$ and cut by restriction enzyme PacI before T4 DNA ligation. GC1::GAD2A-stop and GC1::GAD2-stop were recombined from the pENTR/D-TOPO vector into a pMDC107 vector that contained a GFP tag on the C-terminus (GC1::GAD2 $-G F P$ and $G C 1:: G A D 2-G F P)^{64}$

To create GAD2 complementation driven by a constitutive 35 S promoter, the full-length GAD2 was also amplified using primer set GAD2_forward2 (5'-CACC ATGGTTTTGACAAAAACCGC-3') and GAD2_reverse and cloned into pENTR/ $\mathrm{D}-\mathrm{TOPO}$ vector via directional cloning (Invitrogen), followed by an LR reaction recombinant into pMDC $32^{64}$. For mesophyll specific complementation, GAD2 $\triangle$ with a stop codon was amplified with the GAD2_forward2 and

GAD2_truncated_reverse primer set, and cloned into the pENTR/D-TOPO vector, followed by an LR reaction recombined into the pTOOL5 vector (UAS::GAD2 $\triangle)^{65}$.

For almt $9-2$ complementation, the pART27 binary vector containing the ALMT9 coding sequence ${ }^{21}$ was used for native ALMT9 complementation driven by the $35 \mathrm{~S}$ promoter, and also used as a template for a site-direct mutagenesis PCR to replace F243 and Y245 of ALMT9 with two cysteines (ALMT9F243C/Y245C) using the primer sets: ALMT9_DoubleF (5'-GTTTAGGTGTTAATATGTGTATCTGT CCTATATGGGCTGGAGAGG-3') and ALMT9_DoubleR (5'-CCATATAGGACA GATACACATATTAACACCTAAACTAACACCAGCACC-3').

For GAD2 expression analysis, a $1 \mathrm{~kb}$ sequence upstream of the GAD2 start codon was designated as the GAD2 promoter ( $p G A D 2)$ and amplified using primer set proGAD2_F (5'-ATTTTGAATTTGCGGAGAATCT-3') and proGAD2_R (5'CTTTGTTTCTGTTTAGTGAAAGAGAA-3'). The $p$ GAD2 PCR product was cloned into pCR8/GW/TOPO via TA cloning and recombined via an LR reaction into the pMDC162 vector containing the GUS reporter gene for histochemical assays ${ }^{64}$. The binary vectors, pMDC32, pMDC99, pMDC107, pMDC162, pTOOL5 and pART27 carrying sequence-verified constructs, were transformed into Agrobacterium strain AGL1 for stable transformation in Arabidopsis plants.

Stomatal aperture and density measurement. Soil-grown Arabidopsis (5-6-weekold) were used for stomatal aperture and density measurements. Two-to-three-weekold soybean, broad beans and barley and 5-6-week-old tobacco were used for 
stomatal aperture assays. Epidermal strips from Arabidopsis, soybean, faba bean and tobacco were peeled from abaxial sides of leaves, pre-incubated in stomatal pore measurement buffer containing $10 \mathrm{mM} \mathrm{KCl}, 5 \mathrm{mM}$ L-malic acid, $10 \mathrm{mM} 2$ ethanesulfonic acid (MES) with pH 6.0 by 2-amino-2-(hydroxymethyl)-1,3-propanediol (Tris) under light $\left(200 \mu \mathrm{mol} \mathrm{m}^{-2} \mathrm{~s}^{-1}\right)$ or darkness and transferred into stomatal pore measurement buffer with blind treatments as stated in the figure legend. For barley epidermal stomatal assays, a modified method was used ${ }^{66}$ : the second fully expanded leaf from 2-week-old seedlings was used as experimental material, leaf samples were first detached and bathed in a modified measurement buffer $(50 \mathrm{mM}$ $\mathrm{KCl}, 10 \mathrm{mM}$ MES with $\mathrm{pH} 6.1$ by $\mathrm{KOH})$ under light $\left(150 \mu \mathrm{mol} \mathrm{m}^{-2} \mathrm{~s}^{-1}\right)$ for $1.5 \mathrm{~h}$ or darkness for $1 \mathrm{~h}$, then pre-treated in the same buffer with or without $1 \mathrm{mM} \mathrm{GABA}$ for $0.5 \mathrm{~h}$; after this pre-treatment, samples were incubated in continuous dark, light, light-to-dark or dark-to-light transition for an additional $1 \mathrm{~h}$ as indicated in the figure legend before leaf epidermal strips were peeled for imaging. For Arabidopsis stomatal density measurement, epidermal strips were peeled from abaxial sides of young and mature leaves, three leaves per plants, three plants per genotype. Epidermal strips for both aperture and density measurement were imaged using an Axiophot Pol Photomicroscope (Carl Zeiss) apart from the barley epidermal strips imaged using an Nikon Diaphot 200 Inverted Phase Contrast Microscope (Nikon). Stomatal aperture and density were analyzed using particle analysis (http://rsbweb.nih.gov/ij/).

Stomatal conductance measurement. All stomatal conductance measurements were performed on 5-6-week-old Arabidopsis plants. The stomatal conductance determined by the AP4 Porometer (Delta-T Devices) was calculated based on the mean value from 2-3 leaf recordings per plant (Figs. 3b, $4 c, 5 c, 6 c$, d and 9; Supplementary Fig. 10d, 11d, g and 14c). The time-dependent stomatal conductance, transpiration and photosynthetic rate was recorded using LI-6400XT Portable Photosynthesis System (LI-COR Biosciences) equipped with an Arabidopsis leaf chamber fluorometer (under $150 \mu \mathrm{mol} \mathrm{m}{ }^{-2} \mathrm{~s}^{-1}$ light with $10 \%$ blue light, $150 \mathrm{mmol} \mathrm{s}^{-1}$ flow rate, $400 \mathrm{ppm} \mathrm{CO}$ mixer, $\sim 50 \%$ relative humidity at $22^{\circ} \mathrm{C}$ ) as indicated (Fig. 4d; Supplementary Fig. 8g, h).

ABA measurement. The analysis of Arabidopsis leaf ABA concentration followed a method as described previously ${ }^{67}$. Briefly, $>50 \mathrm{mg}$ of ground fresh leaf samples were used to determine ABA concentration using an Agilent 6410 Series Triple Quad liquid chromatography (LC)-mass spectrometer (MS)/MS, equipped with Agilent 1200 series HPLC (Agilent Technologies) using a Phenomenex C18 column $(75 \mathrm{~mm} \times 4.5 \mathrm{~mm} \times 5 \mu \mathrm{m})$ with a column temperature set at $40{ }^{\circ} \mathrm{C}$. Solvents were nanopure water and acetonitrile, both with $0.05 \%$ acetic acid. Samples were eluted with a linear 15 -min gradient from 10 to $90 \%$ acetonitrile. Compounds were identified by retention times and mass/charge ratio.

Water-deficit drought assay. Plants were germinated on $1 / 2$ MS medium with $0.8 \%$ phytagel for 10 days in short-day conditions $\left(100-120 \mu \mathrm{mol} \mathrm{m}^{-2} \mathrm{~s}^{-1}, 10 \mathrm{~h}\right.$ light/14 h dark) at $22^{\circ} \mathrm{C}$ before being transferred to pots (size 2.5 inch diameter $\times$ 2.25 inch height, LI-COR Bioscience) with soil, containing coco peat/Irish peat (1:1 ratio). Prior to 10-day-old seedling transfer, all pots were weighed on an Ohaus ARA520 Adventurer Balance and soil was aliquoted into the pots within $\pm 0.1 \mathrm{~g}$ between all replicates within an experimental run, randomly placed in growth cabinet and moved every other day in the same environmental conditions stated above. The starting weight varied amongst experimental runs dependent upon soil moisture (from 75 to $78 \mathrm{~g}$ ). The drought assay was performed on 5-6-week-old Arabidopsis plants (Figs. 3, 4g, h and 5; Supplementary Figs. 6a-c, 9 and 11c-e). All plants were well-watered (saturated) the night before the drought assay, but not watered again during the assay. During the drought assay, all plants were randomly moved around once a day to avoid any bias of uneven light distribution or air flow within the cabinet that may differentially affect water loss.

At each sampling point, fresh weight of 2-3 leaves per plant was determined on an Ohaus Explorer E02140 balance (in Fig. 3a, Supplementary Figs. $6 \mathrm{c}$ and 11c, this occurred immediately after the rest of the leaf rosette was snap frozen in liquid nitrogen for later GABA measurement). Sampled leaves were then rehydrated to full turgid weight in ultrapure water overnight and measured after surface water was dried with paper towel. Dry weight was determined at $65^{\circ} \mathrm{C}$ for 1 day. Leaf RWC was calculated as (Figs. 3c, $4 \mathrm{~g}$ and $5 \mathrm{~d}$; Supplementary Figs. 6a and 11e)

$$
\text { RWC }=\frac{\text { Fresh weight }- \text { Dry weight }}{\text { Turgid weight }- \text { Dry weight }} \times 100 \%
$$

At each sampling point, fresh soil weight of the whole pot (Mwet) and dry soil weight after drying the soil (Mdry) at $105^{\circ} \mathrm{C}$ for 3 days was measured using an Ohaus ARA520 Adventurer Balance (Supplementary Fig. 6b). Gravimetric soil water content $(\theta \mathrm{g})$ of the whole soil in the pots was calculated as

$$
\theta \mathrm{g}=\frac{\text { Mwet }- \text { Mdry }}{\text { Mdry }}
$$

Leaf feeding assay. The stomatal conductance, transpiration and photosynthetic rate of the detached leaf feeding assay was recorded using either a LCpro-SD Portable Photosynthesis System (ADC Bioscientific) with $350 \mu \mathrm{mol} \mathrm{m}^{-2} \mathrm{~s}^{-1}$ light, $200 \mu \mathrm{mols} \mathrm{s}^{-1}$ flow rate and $400 \mathrm{ppm} \mathrm{CO}_{2}$ at $22^{\circ} \mathrm{C}$ (Supplementary Fig. 1c, d) or
LI-COR LI-6400XT (LI-COR Biosciences) with $200 \mu \mathrm{mol} \mathrm{m}{ }^{-2} \mathrm{~s}^{-1}$ light, 150 $\mu$ mols s ${ }^{-1}$ flow rate and $400 \mathrm{ppm} \mathrm{CO}_{2}$ at $22^{\circ} \mathrm{C}$ (Figs. $2 \mathrm{c}$ and $8 \mathrm{~b}, \mathrm{~d}$, f; Supplementary Figs. 2a, b and 17a, b, d, e, g, h). The detached leaf was fed with artificia xylem sap solution modified as described ${ }^{68}$, containing $1 \mathrm{mM} \mathrm{KH}_{2} \mathrm{PO}_{4}, 1 \mathrm{mM}$ $\mathrm{K}_{2} \mathrm{HPO}_{4}, 1 \mathrm{mM} \mathrm{CaCl}_{2}, 0.1 \mathrm{mM} \mathrm{MgSO} 4,1 \mathrm{mM} \mathrm{KNO}{ }_{3}, 0.1 \mathrm{mM} \mathrm{MnSO}_{4}, 1 \mathrm{mM} \mathrm{K}-\mathrm{H}$ malate, pH $6.0(\mathrm{KOH})$ with or without GABA or muscimol supplement as indicated, detached leaves were pre-fed under $150 \mu \mathrm{mol} \mathrm{m}{ }^{-2} \mathrm{~s}^{-1}$ light to allow the uptake of treatments for 45-60 min before recording. iWUE and WUE were calculated based on the equation as described in ref. ${ }^{27}$.

GABA measurement. GABA concentration was determined using ultra performance LC (UPLC) as described previously ${ }^{36}$. Briefly, GABA was extracted from samples using $10 \mathrm{mM}$ sodium acetate and derivatized with the AccQ Tag Ultra Derivatization Kit (Waters). Chromatographic analysis of GABA was performed on an Acquity UPLC System (Waters) with a Cortecs or Phenomenex UPLC C18 column $(1.6 \mu \mathrm{m}, 2.1 \times 100 \mathrm{~mm})$. The gradient protocol for amino acids analysis was used to measure GABA with mobile solvents AccQ Tag Ultra Eluents A and B (Waters). Standard GABA solution was used for calibration ranging from 0 to 150 $\mu \mathrm{M}$. The results were analyzed by Empower chromatography software version 3 (Waters)

GUS histochemical staining assays. A GUS histochemical assay was performed using the methods described previously ${ }^{69}$. Three-to-four-week-old transgenic pGAD2::GUS plants were stained in buffer containing $50 \mathrm{mM} \mathrm{Na}$ phosphate $\mathrm{pH}=$ 7.0, $10 \mathrm{mM}$ EDTA, $2 \mathrm{mM}$ potassium ferrocyanide, $2 \mathrm{mM}$ potassium ferricyanide, $0.1 \%(\mathrm{v} / \mathrm{v})$ Triton X-100 and $0.1 \%(\mathrm{w} / \mathrm{v}) \mathrm{X}$-Gluc (5-bromo-4-chloro-3-indolyl $\beta$-D glucuronide) during a $1.5 \mathrm{~h}$ incubation at $37^{\circ} \mathrm{C}$ in the dark. The stained plants were destained in $70 \%$ ethanol. GUS-stained plants were imaged using an Axiophot Pol Photomicroscope (Carl Zeiss).

Fluorescence microscopy. The fluorescence of fluorescent proteins in transgenic $\operatorname{gad} 2-1 / G C 1:: G A D 2 \Delta-G F P, \operatorname{gad} 2-1 / G C 1:: G A D 2-G F P$ and WT/GC1::GAD2 $-G F P$ plants was imaged by confocal laser scanning microscopy using a Zeiss Axioskop 2 mot plus LSM5 PASCAL and argon laser (Carl Zeiss). Sequential scanning and laser excitation was used to capture fluorescence via the LSM5 PASCAL from GFP (excitation $=488 \mathrm{~nm}$, emission band-pass $=505-530 \mathrm{~nm}$ ), chlorophyll autofluorescence $($ excitation $=543 \mathrm{~nm}$, emission long-pass $=560 \mathrm{~nm}$ ). The fluorescence of fluorescent proteins in the mesophyll protoplasts of transgenic almt9-2 complementation lines and $N$. benthamiana (Supplementary Fig 16c, d) was imaged using Nikon A1R Laser Scanning Confocal with DS-Ril CCD camera. Sequential scanning and laser excitation was used to capture fluorescence via the Nikon A1R Laser Scanning Confocal from GFP (excitation $=488 \mathrm{~nm}$, emission $=$ $525-575 \mathrm{~nm})$, chlorophyll autofluorescence $($ excitation $=561 \mathrm{~nm}$, emission $=$ 595-645 nm)

Reverse transcriptional PCR. Reverse transcriptional PCR was determined by PCR amplification on cDNA synthesized from RNA extracted from plants as indicated. PCR amplified GAD2, Actin2, GFP, GAD2 mRNA, UAS::GAD2 2 and ALMT9 using Phire Hot Start II DNA Polymerase (Invitrogen) with primer sets:

GAD2_rt_F (5'-ACGTGATGGATCCAGACAAAG- $\left.3^{\prime}\right)$ and

GAD2_rt-R (5'-TACATTTTCCGCGATCCCT-3');

Actin2_rt_F (5'-CAAAGGCCAACAGAGAGAAGA-3 $\left.{ }^{\prime}\right)$ and

Actin2_rt_R ( $5^{\prime}$-CTGTACTTCCTTTCAGGTGGTG-3');

GFP_rt_F (5'-GGAGTTGTCCCAATTCTTGTT-3') and

GFP_rt_R (5'-CGCCAATTGGAGTATTTTGT-3');

GAD2mRNA_rt_F (5'-ACGTGATGGATCCAGACAAAG- $\left.3^{\prime}\right)$ and

GAD2mRNA_rt_R (5'-TCTTCATTTCCACACAAAGGC-3');

UAS_GAD2_rt_F $\left(5^{\prime}\right.$-TCACTCTCAATTTCTCCAAGG- $\left.3^{\prime}\right)$ and

UAS_GAD2_rt_R (5'-CGGCAACAGGATTCAATCTTAAG-3');

ALMT9_rt_F (5'-AATACTCGAGAAACGGGAGAG-3') and

ALMT9_rt_R (5'-CATCCCAAAACACCTACGAAT-3').

Quantitative real-time PCR analysis. Quantitative reverse transcription PCR was performed using KAPA SYBR FAST ABI PRISM kit (Kapa Biosystems) using a QuantStudio $^{\text {TM }}$ 12K Flex Real-Time PCR System (Thermo Fisher Scientific) to determine the expression levels of GAD1, GAD2, GAD3, GAD4, GAD5, GABA-T, ALMT9, ALMT12, RD29A and RD22 genes with primer sets:

GAD1_qF (5'-TCTCAAAGGACGAGGGAGTG-3') and

GAD1_qR ( $5^{\prime}$-AACCACACGAAGAACAGTGATG-3');

GAD2_qF (5'-GTCTCAAAGGACCAAGGAGTG-3') and

GAD2_qR (5'-CATCGGCAGGCATAGTGTAA- $\left.3^{\prime}\right)$;

GAD3_qF (5'-CCGTTAGTGGCGTTTTCTCT-3') and

GAD3_qR (5'-TCTCTTTGCGTCTCCTCTGG-3');

GAD4_qF (5'-GTGTTCCGTTAGTGGCGTT- $\left.3^{\prime}\right)$ and

GAD4_qR (5'GTCTCCTCTGGCGTCTTCTT-3');

GAD5_qF (5'-TCAACCCACTTTCACTCTCA- $\left.3^{\prime}\right)$ and

GAD5_qR (5'-TTCCTTCTCTTAGCCTCCTT-3');

GABA-T_qF (5'-AGGCAGCACCTGAGAAGAAA-3') and

GABA-T_qR (5'-GGAGTGATAAAACGGCAAGG-3'); 
ALMT9_qF (5'-CAGAGAGTGGGCGTAGAAGG-3') and ALMT9_qR (5'-GGATTTGAAGGCGTAGATTGG-3'); ALMT12_qF (5'-TTGACGGAACTCGCAGATAG-3') and ALMT12_qR (5'-CGATGGAGGTTAGAGCCAAG-3'); RD29A_qF (5'-AAACGACGACAAAGGAAGTG-3') and RD29A_qR (5'-ACCAAACCAGCCAGATGATT-3'); RD22_qF (5'-AGGGCTGTTTCCACTGAGG-3') and RD22_qR (5'- CACCACAGATTTATCGTCAGACA-3'). Expression levels of each gene was normalised to three control genes-Actin2, $E F 1 \alpha$ and $G A P D H-A$-that were amplified with primer sets: Actin2_qF (5'-TGAGCAAAGAAATCACAGCACT-3') and Actin2_qR (5'-CCTGGACCTGCCTCATCATAC-3'); EF1a_qF (5'-GACAGGCGTTCTGGTAAGGAG-3') and EF1a_qR (5'-GCGGAAAGAGTTTTGATGTTCA-3'); GAPDH-A_qF (5'-TGGTTGATCTCGTTGTGCAGGTCTC-3') and GAPDH-A_qR (5'-GTCAGCCAAGTCAACAACTCTCTG-3').

Reporting summary. Further information on research design is available in the Nature Research Reporting Summary linked to this article.

\section{Data availability}

Sequence data used in this paper can be found in The Arabidopsis Information Resource database (https://www.arabidopsis.org/) under the following accessions: GAD1 (At5g17330), GAD2 (At1g65960), GAD3 (At2g02000), GAD4 (At2g02010), GAD5 (At3g17760), GABA-T (At3g22200), ALMT9 (At3g18440), ALMT12 (At4g17970), RD29A (At5g52310) and RD22 (At5g25610). Other data that support the findings of this study are available from the corresponding author upon request. Source Data are provided with this paper.

Received: 19 December 2019; Accepted: 4 February 2021; Published online: 29 March 2021

\section{References}

1. Keenan, T. F. et al. Increase in forest water-use efficiency as atmospheric carbon dioxide concentrations rise. Nature 499, 324 (2013).

2. Papanatsiou, M. et al. Optogenetic manipulation of stomatal kinetics improves carbon assimilation, water use, and growth. Science 363, 1456-1459 (2019).

3. Hetherington, A. M. \& Woodward, F. I. The role of stomata in sensing and driving environmental change. Nature 424, 901 (2003).

4. Shimazaki, K.-i, Doi, M., Assmann, S. M. \& Kinoshita, T. Light regulation of stomatal movement. Annu. Rev. Plant Biol. 58, 219-247 (2007).

5. Sussmilch, F. C., Schultz, J., Hedrich, R. \& Roelfsema, M. R. G. Acquiring control: the evolution of stomatal signalling pathways. Trends Plant Sci. 24, 342-351 (2019).

6. Kim, T.-H., Böhmer, M., Hu, H., Nishimura, N. \& Schroeder, J. I. Guard cell signal transduction network: advances in understanding abscisic acid, $\mathrm{CO}_{2}$, and $\mathrm{Ca}^{2+}$ signaling. Annu. Rev. Plant Biol. 61, 561-591 (2010).

7. Murata, Y., Mori, I. C. \& Munemasa, S. Diverse stomatal signaling and the signal integration mechanism. Annu. Rev. Plant Biol. 66, 369-392 (2015).

8. Melotto, M., Underwood, W., Koczan, J., Nomura, K. \& He, S. Y. Plant stomata function in innate immunity against bacterial invasion. Cell 126, 969-980 (2006).

9. Liu, Y. et al. Anion channel SLAH3 is a regulatory target of chitin receptorassociated kinase PBL27 in microbial stomatal closure. eLife 8, e44474 (2019).

10. Žárský, V. Signal transduction: GABA receptor found in plants. Nat. Plants 1, 15115 (2015).

11. Owens, D. F. \& Kriegstein, A. R. Is there more to GABA than synaptic inhibition? Nat. Rev. Neurosci. 3, 715 (2002).

12. Bouche, N. \& Fromm, H. GABA in plants: just a metabolite? Trends Plant Sci. 9, 110-115 (2004).

13. Kinnersley, A. M. \& Turano, F. J. Gamma aminobutyric acid (GABA) and plant responses to stress. Crit. Rev. Plant Sci. 19, 479-509 (2000).

14. Ramesh, S. A., Tyerman, S. D., Gilliham, M. \& Xu, B. $\gamma$-Aminobutyric acid (GABA) signalling in plants. Cell Mol. Life Sci. 74, 1577-1603 (2017).

15. Bouché, N., Lacombe, B. \& Fromm, H. GABA signaling: a conserved and ubiquitous mechanism. Trends Cell Biol. 13, 607-610 (2003).

16. Bown, A. W. \& Shelp, B. J. Plant GABA: not just a metabolite. Trends Plant Sci. 21, 811-813 (2016).

17. Michaeli, S. \& Fromm, H. Closing the loop on the GABA shunt in plants: are GABA metabolism and signaling entwined? Front. Plant Sci. 6, 419 (2015).

18. Ramesh, S. A. et al. GABA signalling modulates plant growth by directly regulating the activity of plant-specific anion transporters. Nat. Commun. 6, 7879 (2015).
19. Gilliham, M. \& Tyerman, S. D. Linking metabolism to membrane signaling: the GABA-malate connection. Trends Plant Sci. 21, 295-301 (2016).

20. Meyer, S. et al. AtALMT12 represents an R-type anion channel required for stomatal movement in Arabidopsis guard cells. Plant J. 63, 1054-1062 (2010)

21. De Angeli, A., Zhang, J., Meyer, S. \& Martinoia, E. AtALMT9 is a malateactivated vacuolar chloride channel required for stomatal opening in Arabidopsis. Nat. Commun. 4, 1804 (2013).

22. Eisenach, C. et al. ABA-Induced stomatal closure involves ALMT4, a phosphorylation-dependent vacuolar anion channel of Arabidopsis. Plant Cell 29, 2552-2569 (2017).

23. Hauser, F., Li, Z., Waadt, R. \& Schroeder, J. I. SnapShot: abscisic acid signaling. Cell 171, 1708-1708 (2017).

24. Peiter, E. et al. The vacuolar $\mathrm{Ca}^{2+}$-activated channel TPC1 regulates germination and stomatal movement. Nature 434, 404 (2005).

25. Meidner, H. Measurements of stomatal aperture and responses to stimuli. in Stomatal Physiology (ed. Jarvis, P. D. \& Mansfield, T. A.) 25-28 (Cambridge University Press, 1981).

26. Mori, I. C. et al. CDPKs CPK6 and CPK3 function in ABA regulation of guard cell S-type anion-and $\mathrm{Ca}^{2+}$-permeable channels and stomatal closure. PLoS Biol. 4, 1749-1762 (2006).

27. Leakey, A. D. et al. Water use efficiency as a constraint and target for improving the resilience and productivity of $\mathrm{C}_{3}$ and $\mathrm{C}_{4}$ crops. Annu. Rev. Plant Biol. 70, 781-808 (2019).

28. Desikan, R. et al. Hydrogen peroxide and nitric oxide signalling in stomatal guard cells. J. Exp. Bot. 55, 205-212 (2004).

29. Mekonnen, D. W., Flügge, U.-I. \& Ludewig, F. Gamma-aminobutyric acid depletion affects stomata closure and drought tolerance of Arabidopsis thaliana. Plant Sci. 245, 25-34 (2016).

30. Espinoza, C. et al. Interaction with diurnal and circadian regulation results in dynamic metabolic and transcriptional changes during cold acclimation in Arabidopsis. PLoS ONE 5, el4101 (2010).

31. Zik, M., Arazi, T., Snedden, W. A. \& Fromm, H. Two isoforms of glutamate decarboxylase in Arabidopsis are regulated by calcium/calmodulin and differ in organ distribution. Plant Mol. Biol. 37, 967-975 (1998).

32. Yang, Y., Costa, A., Leonhardt, N., Siegel, R. S. \& Schroeder, J. I. Isolation of a strong Arabidopsis guard cell promoter and its potential as a research tool. Plant Methods 4, 6 (2008).

33. Akama, K. \& Takaiwa, F. C-terminal extension of rice glutamate decarboxylase (OsGAD2) functions as an autoinhibitory domain and overexpression of a truncated mutant results in the accumulation of extremely high levels of GABA in plant cells. J. Exp. Bot. 58, 2699-2707 (2007).

34. Lehmann, S. et al. In planta function of compatible solute transporters of the AtProT family. J. Exp. Bot. 62, 787-796 (2011).

35. Meyer, A., Eskandari, S. \& Grallath, S. Rentsch D. AtGAT1, a high affinity transporter for $\gamma$-aminobutyric acid in Arabidopsis thaliana. J. Biol. Chem. 281, 7197-7204 (2006).

36. Ramesh, S. A. et al. Aluminium-activated malate transporters can facilitate GABA transport. Plant Cell 30, 1147-1164 (2018).

37. Martí, M. C., Stancombe, M. A. \& Webb, A. Cell-and stimulus-type-specific intracellular-free $\mathrm{Ca}^{2+}$ signals in Arabidopsis thaliana. Plant Physiol. 163, 625-634 (2013).

38. Kowada, T., Maeda, H. \& Kikuchi, K. BODIPY-based probes for the fluorescence imaging of biomolecules in living cells. Chem. Soc. Rev. 44, 4953-4972 (2015)

39. Long, Y., Tyerman, S. D. \& Gilliham, M. Cytosolic GABA inhibits anion transport by wheat ALMT1. New Phytol. 225, 671-678 (2019).

40. Meyer, S. et al. Malate transport by the vacuolar AtALMT6 channel in guard cells is subject to multiple regulation. Plant J. 67, 247-257 (2011).

41. Kovermann, P. et al. The Arabidopsis vacuolar malate channel is a member of the ALMT family. Plant J. 52, 1169-1180 (2007).

42. Negi, J. et al. $\mathrm{CO}_{2}$ regulator SLAC1 and its homologues are essential for anion homeostasis in plant cells. Nature 452, 483-486 (2008).

43. Vahisalu, T. et al. SLAC1 is required for plant guard cell S-type anion channel function in stomatal signalling. Nature 452, 487-491 (2008).

44. Geiger, D. et al. Activity of guard cell anion channel SLAC1 is controlled by drought-stress signaling kinase-phosphatase pair. Proc. Natl Acad. Sci. USA 106, 21425-21430 (2009).

45. Geiger, D. et al. Guard cell anion channel SLAC1 is regulated by CDPK protein kinases with distinct $\mathrm{Ca}^{2+}$ affinities. Proc. Natl Acad. Sci. USA 107, 8023-8028 (2010)

46. Hedrich, R. \& Geiger, D. Biology of SLAC1-type anion channels-from nutrient uptake to stomatal closure. New Phytol. 216, 46-61 (2017).

47. Takahashi, Y. et al. MAP3Kinase-dependent SnRK2-kinase activation is required for abscisic acid signal transduction and rapid osmotic stress response. Nat. Commun. 11, 1-12 (2020).

48. Chen, Z.-H. et al. Systems dynamic modeling of the stomatal guard cell predicts emergent behaviors in transport, signaling, and volume control. Plant Physiol. 159, 1235-1251 (2012). 
49. Hills, A., Chen, Z.-H., Amtmann, A., Blatt, M. R. \& Lew, V. L. OnGuard, a computational platform for quantitative kinetic modeling of guard cell physiology. Plant Physiol. 159, 1026-1042 (2012).

50. Takahashi, F. et al. A small peptide modulates stomatal control via abscisic acid in long-distance signalling. Nature 556, 235 (2018).

51. Kuromori, T., Seo, M. \& Shinozaki, K. ABA transport and plant water stress responses. Trends Plant Sci. 23, 513-522 (2018).

52. Sharma, T., Dreyer, I., Kochian, L. \& Piñeros, M. A. The ALMT family of organic acid transporters in plants and their involvement in detoxification and nutrient security. Front. Plant Sci. 7, 1488 (2016).

53. Zhang, J. et al. Identification of a probable pore-forming domain in the multimeric vacuolar anion channel AtALMT9. Plant Physiol. 163, 830-843 (2013).

54. Marvin, J. S. et al. A genetically encoded fluorescent sensor for in vivo imaging of GABA. Nat. Methods 16, 763-770 (2019).

55. Fromm, H. GABA signaling in plants: targeting the missing pieces of the puzzle. J. Exp. Bot. 71, 6238-6245 (2020)

56. Liu, J., Zhou, M., Delhaize, E. \& Ryan, P. R. Altered expression of a malatepermeable anion channel, OsALMT4, disrupts mineral nutrition. Plant Physiol. 175, 1745-1759 (2017).

57. Balzergue, C. et al. Low phosphate activates STOP1-ALMT1 to rapidly inhibit root cell elongation. Nat. Commun. 8, 15300 (2017).

58. Jie, Y. et al. An InDel in the promoter of Al-activated malate transporter 9 selected during tomato domestication determines fruit malate contents and aluminum tolerance. Plant Cell 29, 2249-2268 (2017).

59. Palanivelu, R., Brass, L., Edlund, A. F. \& Preuss, D. Pollen tube growth and guidance is regulated by POP2, an Arabidopsis gene that controls GABA levels. Cell 114, 47-59 (2003).

60. Domingos, P. et al. Molecular and electrophysiological characterization of anion transport in Arabidopsis thaliana pollen reveals regulatory roles for $\mathrm{pH}$, $\mathrm{Ca}^{2+}$ and GABA. New Phytol. 223, 1353-1371 (2019).

61. Svozil, J., Gruissem, W. \& Baerenfaller, K. Proteasome targeting of proteins in Arabidopsis leaf mesophyll, epidermal and vascular tissues. Front. Plant Sci. 6, 376 (2015).

62. Prusko, K. Identifizierung Cis-Regulatorischer Elemente der Transkriptionskontrolle in Photosynthetisch Aktiven Blattzellen von Arabidopsis thaliana, $\mathrm{PhD}$ thesis (Heinrich-Heine-Universität Düsseldorf, 2010).

63. Conn, S. J. et al. Protocol: optimising hydroponic growth systems for nutritional and physiological analysis of Arabidopsis thaliana and other plants. Plant Methods 9, 4 (2013).

64. Curtis, M. D. \& Grossniklaus, U. A gateway cloning vector set for highthroughput functional analysis of genes in planta. Plant Physiol. 133, 462-469 (2003).

65. Møller, I. S. et al. Shoot $\mathrm{Na}^{+}$exclusion and increased salinity tolerance engineered by cell type-specific alteration of $\mathrm{Na}^{+}$transport in Arabidopsis. Plant Cell 21, 2163-2178 (2009).

66. Shen, L. et al. Measuring stress signaling responses of stomata in isolated epidermis of graminaceous species. Front. Plant Sci. 6, 533 (2015).

67. Speirs, J., Binney, A., Collins, M., Edwards, E. \& Loveys, B. Expression of ABA synthesis and metabolism genes under different irrigation strategies and atmospheric VPDs is associated with stomatal conductance in grapevine (Vitis vinifera L. cv Cabernet Sauvignon). J. Exp. Bot. 64, 1907-1916 (2013).

68. Wilkinson, S. \& Davies, W. J. Xylem sap pH increase: a drought signal received at the apoplastic face of the guard cell that involves the suppression of saturable abscisic acid uptake by the epidermal symplast. Plant Physiol. 113, 559-573 (1997).

69. $\mathrm{Xu}, \mathrm{B}$. et al. A calmodulin-like protein regulates plasmodesmal closure during bacterial immune responses. New Phytol. 215, 77-84 (2017).

\section{Acknowledgements}

The authors would like to thank Dr Katja Bärenfaller from the University of Zurich for providing JR11-2 (Col-0) seeds; Prof. Zhonghua Chen from Western Sydney University for assisting with barley epidermal assays; Dr Cornelia Eisenach from the University of Zurich for providing ALMT9 constructs, almt12 and almt9 seeds and Prof. Stephen D Tyerman, Prof. Enrico Martinoia and Dr Alexis De Angeli for valuable discussions. This work was funded by Waite Research Institute, funding to M.G. and B.X., ARC Discovery grants DP170104384 and DP21012828 to M.G. and R.H. and ARC Centre of Excellence (CE14010008) and Grains Research and Development Corporation funding (UWA00173) to M.G. Author X.F. was supported by supported by a Chinese Scholarship Council. X.Z. was supported by a Chinese Scholarship Council Travelling Fellowship.

\section{Author contributions}

B.X. constructed all materials and performed all experiments except the following: Y.L. generated almt9/almt12 mutants and performed experiments on stomatal aperture assays treated with hydrogen peroxide and calcium and of almt12 and almt9/12 mutants. X.F. generated almt 9 complementation lines, performance aperture and conductance measurement of complementation plants. X.Z. performed all non-Arabidopsis aperture measurements, except for barley performed by N.S. Author L.C. performed GABA measurements supervised by M.O. Author A.B. performed ABA quantification supervised by E.J.E. Author J.H. acquired images used in Fig. 1. M.G., B.X. and R.H. conceived the research. B.X. drafted all figures. M.G. and B.X. drafted the manuscript. All authors provided edits.

\section{Competing interests}

The authors declare no competing interests.

\section{Additional information}

Supplementary information The online version contains supplementary material available at https://doi.org/10.1038/s41467-021-21694-3.

Correspondence and requests for materials should be addressed to M.G.

Peer review information Nature Communications thanks the anonymous reviewers for their contribution to the peer review of this work. Peer review reports are available.

Reprints and permission information is available at http://www.nature.com/reprints

Publisher's note Springer Nature remains neutral with regard to jurisdictional claims in published maps and institutional affiliations.

\begin{abstract}
(c) (i) Open Access This article is licensed under a Creative Commons BY Attribution 4.0 International License, which permits use, sharing, adaptation, distribution and reproduction in any medium or format, as long as you give appropriate credit to the original author(s) and the source, provide a link to the Creative Commons license, and indicate if changes were made. The images or other third party material in this article are included in the article's Creative Commons license, unless indicated otherwise in a credit line to the material. If material is not included in the article's Creative Commons license and your intended use is not permitted by statutory regulation or exceeds the permitted use, you will need to obtain permission directly from the copyright holder. To view a copy of this license, visit http://creativecommons.org/ licenses/by/4.0\%.
\end{abstract}

(C) The Author(s) 2021 